\title{
Resolutions and Cohomologies of Toric Sheaves. The affine case
}

\author{
Markus Perling*
}

June 2011

Dedicated to Günther Trautmann on the occasion of his 70th birthday.

\begin{abstract}
We study equivariant resolutions and local cohomologies of toric sheaves for affine toric varieties, where our focus is on the construction of new examples of indecomposable maximal Cohen-Macaulay modules of higher rank. A result of Klyachko states that the category of reflexive toric sheaves is equivalent to the category of vector spaces together with a certain family of filtrations. Within this setting, we develop machinery which facilitates the construction of minimal free resolutions for the smooth case as well as resolutions which are acyclic with respect to local cohomology functors for the general case. We give two main applications. First, over the polynomial ring, we determine in explicit combinatorial terms the $\mathbb{Z}^{n}$-graded Betti numbers and local cohomology of reflexive modules whose associated filtrations form a hyperplane arrangement. Second, for the non-smooth, simplicial case in dimension $d \geq 3$, we construct new examples of indecomposable maximal Cohen-Macaulay modules of rank $d-1$.
\end{abstract}

\section{Contents}

1 Introduction $\quad 2$

2 Graded modules and poset representations 5

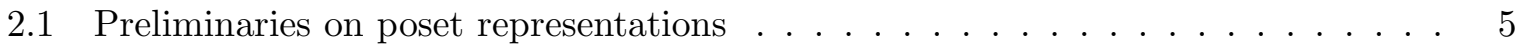

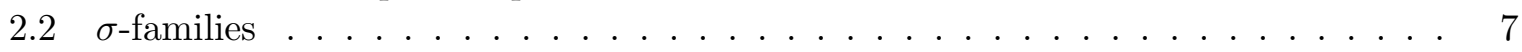

2.3 Matlis duality, injective and projective modules . . . . . . . . 8

2.4 Minimizing projective and injective resolutions . . . . . . . . . . . 10

2.5 Homogeneous coordinates and divisorial modules . . . . . . . . . . . . 11

3 Resolutions $\mathbf{1 3}$

$3.1 \mathrm{lcm}-$ and gcd-lattices . . . . . . . . . . . . . . . . 14

$3.2 \mathrm{lcm}$-lattices of reflexive modules . . . . . . . . . . . . . . 16

3.3 Combinatorially finite modules . . . . . . . . . . . . . . 17

3.4 Using lcm- and gcd-lattices to compute resolutions . . . . . . . . . . . 18

3.5 Reflexive modules and resolutions of vector space arrangements . . . . . . . . . 20

3.6 Duality of resolutions and local cohomology . . . . . . . . . . . . 22

3.7 Computing local cohomologies . . . . . . . . . . . . . . 2 25

${ }^{*}$ Fakultät für Mathematik Ruhr-Universität Bochum Universitätsstraße 15044780 Bochum, Germany, Markus.Perling@rub.de 
4 Hyperplane arrangements 26

4.1 Hyperplane modules . . . . . . . . . . . . . . . . . . . . 26

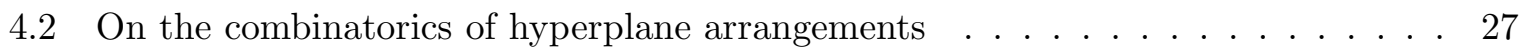

4.3 Free resolutions . . . . . . . . . . . . . . . . . . . 28

4.4 Local cohomology . . . . . . . . . . . . . . . . . . . . . . . 31

5 Codivisorial resolutions and maximal Cohen-Macaulay modules 33

5.1 Homogeneous coordinates and local cohomology . . . . . . . . . . . . 33

5.2 Vector space arrangements with trivial intersections . . . . . . . . . . . . . 34

\section{References}

\section{Introduction}

In this article we want to study equivariant resolutions and local cohomologies of toric sheaves for affine toric varieties. Our aim is to identify good invariants and to set up the machinery which allows to determine explicitly Betti and Bass numbers and local cohomologies in many interesting cases, as well as to construct new examples of indecomposable maximal CohenMacaulay modules of rank greater than one. Let $M$ and $N$ denote the character and co-character group of a $d$-dimensional algebraic torus over some field $\mathbb{K}$, and $\sigma_{M} \subseteq M$ a subsemigroup corresponding to a strictly convex rational polyhedral cone $\sigma \subseteq N \otimes_{\mathbb{Z}} \mathbb{R}$ such that $\mathbb{K}\left[\sigma_{M}\right]$ is the coordinate ring of a normal affine toric variety $U_{\sigma}=\operatorname{spec}\left(\mathbb{K}\left[\sigma_{M}\right]\right)$. Equivariant sheaves on $U_{\sigma}$ then are equivalent to $M$-graded $\mathbb{K}\left[\sigma_{M}\right]$-modules. Our main goal is to study $M$-graded local cohomologies of such modules in terms of $M$-graded acyclic resolutions. For this, we proceed in two steps, where we start with the case of free and injective $\mathbb{Z}^{n}$-graded resolutions over the polynomial ring $S=\mathbb{K}\left[x_{1}, \ldots, x_{n}\right]$ (our results are related to earlier work on injective resolutions such as [Mil00, [HM03, [HM05]). For the second step, we can assume by a construction of Cox that $\mathbb{K}\left[\sigma_{M}\right]$ is a subring of some $S$, where $n$ denotes the number of one-dimensional cones in $\sigma$. More precisely, we can identify $\mathbb{K}\left[\sigma_{M}\right]$ with the degree-zero part of $S$ with respect to its grading by a certain abelian group $A$ (see subsection 2.5). The $A$-grading on $S$ is compatible with its natural $\mathbb{Z}^{n}$-grading in the sense that by taking degree zero with respect to the $A$-grading, we obtain an essentially surjective functor from the category of $\mathbb{Z}^{n}$-graded $S$-modules to the category $M$-graded $\mathbb{K}\left[\sigma_{M}\right]$-modules (see [BC94] Prop. 4.17). In particular, $\mathbb{Z}^{n}$-graded injective resolutions descend to $M$-graded resolutions which are acyclic with respect to local cohomology with monomial support.

The subject of $M$-graded $\mathbb{K}\left[\sigma_{M}\right]$-modules has been well studied. See the book MS04 for a general overview on the subject and the articles [Röm01, Yan01, Yan03, Tch07, for works which share some common features with ours. The new aspect we bring into this subject is the following. It was observed by Klyachko [Kly90, [Kly91 that the class of $M$-graded $\mathbb{K}\left[\sigma_{M}\right]$ modules is a natural extension of toric geometry in terms of linear algebra. Therefore these modules exhibit a rich combinatorial content, as is made explicit by the following theorem for the case of reflexive modules.

Theorem 1.1 (Kly90, Kly91, see also Per04]): The category of finitely-generated, M-graded reflexive $\mathbb{K}\left[\sigma_{M}\right]$-modules is equivalent to the category of vector spaces $\mathbf{E}$ endowed with $n$ filtrations $0 \subseteq \cdots \subseteq E^{k}(i) \subseteq E^{k}(i+1) \subseteq \cdots \subseteq \mathbf{E}$ for $k \in[n]$ which are full in the sense that $E^{k}(i)=0$ for $i<<0$ and $E^{k}(i)=\mathbf{E}$ for $i>>0$.

Note that here the set $[n]=\{1, \ldots, n\}$ is identified with the set of rays of the cone $\sigma$ (see also our notations and conventions below). For the case of $\mathbb{Z}^{n}$-graded $S$-modules, a standard method to extract combinatorial content from such a module is to consider certain finite, adapted subsets of $\mathbb{Z}^{n}$, so-called lcm-lattices. Here, we consider $\mathbb{Z}^{n}$ as a poset by setting $\left(c_{1}, \ldots, c_{n}\right) \leq\left(c_{1}^{\prime}, \ldots, c_{n}^{\prime}\right)$ 
iff $c_{k} \leq c_{k}^{\prime}$ for every $k \in[n]$. The lcm of any two elements $\underline{c}, \underline{c}^{\prime} \in \mathbb{Z}^{n}$ is defined by taking the componentwise maximum. Originally, lcm-lattices have been introduced in GPW99] for monomial ideals. A definition for lcm-lattices of general $\mathbb{Z}^{n}$-graded $S$-modules has been proposed in [CT03. In general, one could consider the defining properties for an (admissible) lcm-lattice of a graded module $E$ that it is closed under taking lcms in $\mathbb{Z}^{n}$ and that it contains all possible degrees of nonzero graded Betti numbers of $E$. Theorem 1.1 connects to this by giving rise to a very nice interpretation for the lcm-lattice of a reflexive module. Namely, let $E$ be such a module with associated filtrations as in Theorem 1.1 and and denote $\mathcal{V}$ the vector space arrangement in $\mathbf{E}$ generated by the intersections of the vector spaces $E^{k}(i)$. Then we observe that the mapping $\mathcal{V} \rightarrow \mathbb{Z}^{n}$ given by

$$
X \mapsto \underline{i}^{X}=\left(i_{1}^{X}, \ldots, i_{n}^{X}\right),
$$

where $i_{k}^{X}=\min \left\{i \mid X \subseteq E^{k}(i)\right\}$ embeds $\mathcal{V}$ as a poset into $\mathbb{Z}^{n}$. The image of this map then indeed is a minimal admissible lcm-lattice for $E$ (see Proposition 3.12). Now, let a minimal free graded free resolution $0 \rightarrow F_{t} \rightarrow \cdots \rightarrow F_{0} \rightarrow E \rightarrow 0$ be given, then the modules $F_{i}$ as well as the syzygy modules are reflexive as well and to each of these we have associated filtrations. Now, the most important structural observation is that we can transport the notion of free resolutions over to the setting of vector spaces arrangements. Forgetting about the modules and only considering the induced maps of the underlying vector space arrangements, we obtain an exact sequence of vector space arrangements:

$$
0 \longrightarrow \mathcal{F}_{t} \longrightarrow \cdots \longrightarrow \mathcal{F}_{0} \longrightarrow \mathcal{V} \longrightarrow 0
$$

Here, the $\mathcal{F}_{i}$ denotes the coordinate arrangements associated to the free modules $F_{i}$. Cutting this sequence in short exact pieces $0 \rightarrow \mathcal{V}_{i+1} \rightarrow \mathcal{F}_{i} \rightarrow \mathcal{V}_{i} \rightarrow 0$ (with $\mathcal{V}=\mathcal{V}_{0}$ ), we obtain an iterative construction of $\mathcal{V}_{i}$ as the $i$-th syzygy arrangement of $\mathcal{V}$. As we will see in subsection 3.5. the construction of syzygy arrangements can be done intrinsically in the category of vector space arrangements. Indeed, for reflexive modules with equivalent underlying vector space arrangements, we obtain equivalent free resolutions in the sense that we can identify their nonzero graded Betti numbers via a poset isomorphism between the sets of nonzero graded Betti numbers.

Theorem (3.27): Let E be a $\mathbb{Z}^{n}$-graded, finitely generated, reflexive $S$-module. Then the poset of nonzero graded Betti numbers is determined by the embedding of the poset given by the underlying vector space arrangement $\mathcal{V}$ into $\mathbb{Z}^{n}$ For given $X \in \mathcal{V}$, the corresponding Betti number depends only on $\mathcal{V}$.

The remarkable implication of this theorem is that by our approach we obtain new invariants of $\mathbb{Z}^{n}$-graded modules. Most of this paper will be devoted to setting up machinery to make above construction work and to utilize it for local cohomology computations both over $S$ and over $\mathbb{K}\left[\sigma_{M}\right]$. This will in particular be done by adapting results of Miller [Mil00] to our setting (see subsections 3.6 and 3.7).

To show the versatility of our methods we will present two important applications. The first will be the explicit computation of graded Betti numbers and local cohomology of reflexive $S$-modules whose associated filtrations form hyperplane arrangements. Assume that $E$ is a reflexive $\mathbb{Z}^{n}$-graded $S$-module whose associated filtrations form an essential hyperplane arrangement $\mathcal{H}$. We denote $X \mapsto \underline{c}^{X}$ the poset embedding of $\mathcal{H}$ into $\mathbb{Z}^{n}$. Then the graded Betti numbers are given by $\beta_{i}\left(\underline{c}^{X}\right)$ for $i \geq 0$ and $X \in \mathcal{H}$. We get:

Theorem (4.5): Let $E$ be a reflexive $\mathbb{Z}^{n}$-graded $S$-module whose associated filtrations form an essential hyperplane arrangement $\mathcal{H}$. Then for any $X \in \mathcal{H}$ the associated graded Betti numbers $\beta_{i}\left(\underline{c}^{X}\right)$ are zero unless $\operatorname{dim} X=i+1$. If $\operatorname{dim} X=i+1$, then $\beta_{i}\left(\underline{c}^{X}\right)$ coincides with the beta invariant of $X$. 
Note that the beta invariant is a combinatorial invariant of a ranked poset which can completely be expressed in terms of its Möbius function (see subsection 4.2 for details). For a given $X \in \mathcal{H}$, the beta invariant is the one associated to the hyperplane arrangement $\{X \cap Y \mid Y \in \mathcal{H}\}$ in $X$.

Now consider the local cohomology $H_{x}^{i} E$ of such modules with respect to the maximal homogeneous ideal of $S$ (equivalently, with respect to the fixed point $x=0$ of the standard torus action on the affine space $\mathbf{A}_{\mathbb{K}}^{n}$ ). Given the degrees $\underline{c}^{X}$ as above, then by duality (see subsections 3.6 and 3.7), the graded pieces of $H_{x}^{i} E$ are determined by the gcd-lattice $\mathcal{G}$ (see subsection 3.1) generated by the Bass numbers of $E$, which are given by elements $\left\{\underline{d}^{X} \mid X \in \mathcal{H}\right\}$, where $\underline{d}^{X}=\underline{c}^{X}-(1, \ldots, 1)$ for every $X \in \mathcal{H}$. An element $\underline{c} \in \mathbb{Z}^{n}$ is adjacent to $\underline{d} \in \mathbb{Z}^{n}$ if $\left(H_{x}^{i} E\right)_{\underline{c}}$ is determined by (and therefore coincides with) $\left(H_{x}^{i} E\right)_{\underline{d}}$. The following result shows that the local cohomology of $E$ encodes the whole spectrum of beta invariants of subarrangements of $\mathcal{H}$.

Theorem (4.13): Let $E$ be a reflexive $\mathbb{Z}^{n}$-graded $S$-module whose associated filtrations form an essential hyperplane arrangement $\mathcal{H}$ in $V$. Denote $\mathcal{G}$ the gcd-lattice generated by the degrees of the Bass numbers of $E$. For every $\underline{d} \in \mathcal{G}$ denote $\mathcal{H} \underline{\underline{d}} \subset \mathcal{H}$ the hyperplane arrangement generated by those hyperplanes $H \in \mathcal{H}$ with $\underline{d} \not \leq \underline{d}^{H}, r \underline{d}$ its rank and $\beta \underline{\underline{d}}$ its beta invariant. Then for any $\underline{d} \in \mathcal{G}$ and any $\underline{c} \in \mathbb{Z}^{n}$ adjacent to $\underline{d}$ we have

$$
\operatorname{dim}\left(H_{x}^{i} E\right)_{\underline{c}}= \begin{cases}\beta \underline{\underline{d}} & \text { if } i=n-r \underline{\underline{d}}+1 \\ 0 & \text { else. }\end{cases}
$$

Our second application will be the explicit construction of new examples of $M$-graded maximal Cohen-Macaulay (MCM) modules over $\mathbb{K}\left[\sigma_{M}\right]$ for the case that $\sigma$ is simplicial but not regular. It is well known that there is - up to degree-shift - a one-to-one correspondence between MCM modules of rank one and Weil divisor classes on $\operatorname{spec}\left(\mathbb{K}\left[\sigma_{M}\right]\right)$. In AR89 Auslander and Reiten show that the toric ring $\mathbb{K}[[X, Y, X]]^{\mathbb{Z} / 2 \mathbb{Z}}$ is MCM-finite and that indeed there exists an MCM module of rank two over this ring (see also Yos90 §16). The main result of section 5 will be that this example fits into a general class of examples of MCM modules over simplicial toric rings.

Theorem (5.9): Let $\sigma$ be a simplicial and non-regular cone of dimension $d \geq 3$. Then there exists an indecomposable $M$-graded MCM module of rank $d-1$.

In general, there is even more than one isomorphism class. More precise statements will be given in subsection 5.2 .

As a final remark I want to mention that in a late stage of writing this article Bernd Sturmfels brought to my attention that some very similar ideas have been developed independently in the context of topological data analysis. The theory of multi-dimensional persistence essentially parallels the idea of $\sigma$-families in [Per04 from a topological point of view (e.g. see [CZ09], [Knu08]). It would be interesting to see whether our work might lead to interesting applications in this area.

\section{Acknowledgements.}

For conversations which brought new ideas into this project I want to thank Günther Trautmann, Henning Krause, Ezra Miller, and Vic Reiner.

\section{Overview of the paper.}

Section 2 contains general facts about poset representations and graded modules; most of the material is well-known. In section 3 we will develop our machinery for computing resolutions and local cohomologies of graded modules. The results will be applied in section 4 to compute Betti 
and Bass numbers and the local cohomology of $\mathbb{Z}$-graded reflexive $S$-modules whose associated filtrations generate hyperplane arrangements. Finally, in section 5 , we construct examples of indecomposable MCM-modules of higher rank for the simplicial case.

\section{Notation and general conventions.}

Throughout this text $\mathbb{K}$ shall denote a fixed field. The general setting of this paper is that of finitely generated, normal monoid rings over $\mathbb{K}$, i.e. rings of the form $\mathbb{K}\left[\sigma_{M}\right]$, where $\sigma_{M}=\check{\sigma} \cap M$. Here, $M \cong \mathbb{Z}^{d}$ is identified with the character group of the $d$-dimensional torus $T=\mathbb{G}_{m}^{d}(\mathbb{K})$ and $N$ the dual group of cocharacters. Moreover, $\sigma \subset N_{\mathbb{R}}=N \otimes_{\mathbb{Z}} \mathbb{R}$ denotes a strictly convex rational polyhedral cone and $\check{\sigma} \subset M_{\mathbb{R}}=M \otimes_{\mathbb{Z}} \mathbb{R}$ its dual cone with respect to the standard bilinear pairing $M \times N \rightarrow \mathbb{Z},(m, n) \mapsto m(n)$. Identifying $N$ with twice its dual, we will usually write $n(m)$ rather than $m(n)$. Throughout we will assume that a $d$-dimensional cone $\sigma$ will be fixed. Although we do not assume that $\mathbb{K}$ is algebraically closed, we will make use of well-known standard facts of toric geometry, such as the correspondence between the the faces of $\sigma, T$-orbits on the associated affine toric $\mathbb{K}$-scheme $U_{\sigma}=\operatorname{spec}\left(\mathbb{K}\left[\sigma_{M}\right]\right)$ and $M$-graded prime ideals in $\mathbb{K}\left[\sigma_{M}\right]$. Within the scope of this paper, the relevant results contained in standard references such as Oda88] and [Ful93] are applicable to our setting. For some $n \in \mathbb{N}$ we denote by $[n]$ the ordered set $\{1<\cdots<n\}$. We will assume that $\sigma$ has $n$ rays with primitive vectors $l_{1}, \ldots, l_{n}$. By abuse of notation, we will in general not distinguish between the $l_{i}$ and the rays they generate. Moreover, we will often identify the set $\left\{l_{1}, \ldots, l_{n}\right\}$ with $[n]$.

We will throughout consider graded modules over graded commutative rings. For general reference for general categorial properties of graded rings and modules we refer to [Nv04]. Tensor products and Hom of graded modules will always be considered as graded. In particular, for any commutative ring $R$ graded by some (additive) abelian group $G$ and some $G$-graded $R$-modules $E, F$, the module $\operatorname{Hom}_{R}(E, F)$ will be defined as

$$
\operatorname{Hom}_{R}(E, F):=\bigoplus_{g \in G} \operatorname{Hom}_{R}(E, F)_{g}
$$

with $\operatorname{Hom}_{R}(E, F)_{g}=\operatorname{Hom}_{R}(E(-g), F)_{0}=\operatorname{Hom}_{R}(E, F(g))_{0}$. We will also write $\operatorname{Hom}_{R}^{G}(E, F)$ for $\operatorname{Hom}_{R}(E, F)_{0}$. The graded tensor product of $E \otimes_{R} F$ is considered as graded by setting $\left(E \otimes_{R} F\right)_{g}$ the submodule generated by elements $e \otimes f$ with $e \in E_{h}$ and $f \in F_{g-h}$ for any $h \in G$. Alternatively, if $R$ is a $\mathbb{K}$-algebra, $E \otimes F$ can be considered as the quotient of the $\mathbb{K}$-vector space $E \otimes_{\mathbb{K}} F$, which is graded by setting $\left(E \otimes_{\mathbb{K}} F\right)_{g}=\bigoplus_{h \in G}\left(E_{h} \otimes_{\mathbb{K}} F_{g-h}\right)$, by the subvector space generated by $r e \otimes f-e \otimes r f$ for $e \in E, f \in F, r \in R$. Note that $E(g) \otimes_{R} F \cong$ $E \otimes_{R} F(g) \cong(E \otimes F)(g)$. We will denote $G$-R-Mod the category of $G$-graded $R$-modules where the morphisms are given by $\operatorname{Hom}_{R}^{G}(E, F)$ for any two $G$-graded modules $E, F$.

\section{Graded modules and poset representations}

We will start in subsection 2.1 with some general remarks on sheaves on posets and preordered sets, respectively. In subsection 2.2 we recapitulate material from [Per04] on $\sigma$-families and add some complementary remarks. In subsections 2.3 and 2.4 we introduce Matlis duality and injective and and projective objects and discuss resolutions. In subsection 2.5] we introduce divisorial and codivisorial modules and resolutions via the homogeneous coordinate ring.

\subsection{Preliminaries on poset representations}

In this work we will make extensive use of $\mathbb{K}$-linear representations of posets as well as limits and colimits of such representations. Therefore it will be useful to have several equivalent formulations for these kind of objects at hand. There is an extensive literature about poset 
representations and their limits of which only a very small part is relevant for us. For a recent survey we refer to Web07. Basic information about limits and colimits can be found e.g. in Eis95, Appendix A6.

Let $\mathcal{P}$ be any preordered set with order relation $\leq$. Recall that a preorder is defined by the same axioms as a partial order, except for the reflexitivity axiom, i.e. there may exist elements $x, y \in \mathcal{P}$ such that $x \leq y$ and $y \leq x$, but $x \neq y$. Then $\mathcal{P}$ in a natural way forms a category: its objects are given by the set underlying $\mathcal{P}$ and the morphisms for $x, y \in \mathrm{Ob}(\mathcal{P})$ are:

$$
\operatorname{Mor}(x, y)= \begin{cases}\text { the pair }(x, y) & \text { if } x \leq y \\ \emptyset & \text { else }\end{cases}
$$

with composition given by $(y, z)(x, y)=(x, z)$ whenever $x \leq y \leq z$. Therefore the pair $(x, x)$ represents the identity morphism for all $x \in \mathcal{P}$.

Definition 2.1: A $\mathbb{K}$-linear representation of $\mathcal{P}$ is a functor from $\mathcal{P}$ to the category of $\mathbb{K}$-vector spaces.

Any such functor $E$ associates to $x \in \mathcal{P}$ a $\mathbb{K}$-vector space $E_{x}$ and to a morphism $x \leq y$ in $\mathcal{P}$ a $\mathbb{K}$-linear homomorphism $E(x, y): E_{x} \rightarrow E_{y}$. It is straightforward to see that the $\mathbb{K}$-linear representations of $\mathcal{P}$ together with their natural transformations form an abelian category.

We can always pass from the preordered set $\mathcal{P}$ to its canonically associated poset $\mathcal{P} / \sim$, where $\sim$ denotes the equivalence relation on $\mathcal{P}$ given by $x \sim y$ iff $x \leq y$ and $y \leq x$. We get:

Lemma 2.2: The category of $\mathbb{K}$-linear representations of $\mathcal{P}$ is equivalent to the category of $\mathbb{K}$-linear representations of $\mathcal{P} / \sim$.

Proof. Let $E$ be a $\mathbb{K}$-linear representation of $\mathcal{P}$. For any $x \sim y$, the morphism $(x, y)$ is an isomorphism in $\mathcal{P}$ and thus $E(x, y)$ is an isomorphism of $\mathbb{K}$-vector spaces whose inverse is $E(y, x)$. In particular, we have $E_{x} \cong E_{y}$ for every pair $x \sim y$. To define a representation of $\mathcal{P} / \sim$, we set $E_{[x]}:=\lim _{\longrightarrow} E_{y}$, where $[x]$ denotes an equivalence class of $\sim$ and the colimit is taken over all elements $y$ in this equivalence class. Then by the naturality of colimits, we obtain a morphism $E([x],[y]): E_{[x]} \rightarrow E_{[y]}$ for any pair $(x, y)$. These morphisms are compatible with composition in $\mathcal{P}$ by the functoriality of colimits.

For the other direction, we can lift representation $E$ of $\mathcal{P} / \sim$ to a representation of $\mathcal{P}$ by setting $E_{x}:=E_{[x]}$ and $E(x, y):=E([x],[y])$. It is straightforward to see that these two functors indeed establish an equivalence of categories.

Remark 2.3: The limit $\lim _{y} E_{y}$ in the proof of Lemma 2.2 should be considered as a "generic" representative for the vector spaces $E_{y}$ with $y$ in one given equivalence class which does not depend on some particular choices. In particular, $\underset{\lim }{\longrightarrow} E_{y}$ is isomorphic to any $E_{y}$.

Remark 2.4: So, strictly speaking, it does not seem necessary to consider preordered sets rather than just posets. However, as we will see later on, from the point of view of toric geometry it will be more natural to first consider preordered sets.

On $\mathcal{P}$ there is defined a topology which is generated by the basis

$$
U(x):=\{y \geq x\}
$$

for all $x \in \mathcal{P}$. Note that the continuous maps between preordered sets then are precisely the order preserving maps.

Proposition 2.5: Let $\mathcal{P}$ be a preordered set. Then there is an equivalence of categories between the categories of representations of $\mathcal{P}$ and of sheaves of $\mathbb{K}$-vector spaces on $\mathcal{P}$. 
Proof. A sheaf of $\mathbb{K}$-vector spaces $\mathcal{E}$ on $\mathcal{P}$ with respect to this topology automatically induces a $\mathbb{K}$-linear representation of $\mathcal{P}$ by setting $E_{x}=\mathcal{E}(U(x))$ for every $x \in \mathcal{P}$ and $E(x, y)$ the restriction morphism $\mathcal{E}(U(x)) \rightarrow \mathcal{E}(U(y))$. On the other hand, for any representation $E$, following GD71 $\S 0.3 .2$, we obtain a presheaf $\mathcal{E}$ on $\mathcal{P}$ by setting $\mathcal{E}\left((U(x)):=E_{x}\right.$ for all $x \in \mathcal{P}$ and $\mathcal{E}(U):=\lim \mathcal{E}(U(x))$ for some open set $U$, where the limit runs over all $x \in U$. Note that the stalk $\mathcal{E}_{x}$ is isomorphic to $\mathcal{E}(U(x))$. Observing that for some $U(x)$ every open cover of $U(x)$ necessarily contains $U(x)$ itself, we can apply the criterion of $\S 0.3 .2 .2$ in [GD71] from which it follows that our presheaf is a sheaf.

\section{$2.2 \quad \sigma$-families}

In this subsection we will recall some material from [Per04]. In [Per04, the general assumption was used that $\mathbb{K}$ is algebraically closed. However, the results relevant for us actually do not depend on any properties of $\mathbb{K}$ and therefore will be stated without any assumptions on $\mathbb{K}$.

We will fix some more notation. Elements of $M$ are denoted $m, m^{\prime}$ etc. if written additively and $\chi(m), \chi\left(m^{\prime}\right)$ etc. if written multiplicatively, i.e. $\chi\left(m+m^{\prime}\right)=\chi(m) \chi\left(m^{\prime}\right)$. Faces of $\sigma$ are denoted by small Greek letters $\rho, \tau$ etc; the face order among faces is denoted $\rho \preceq \tau$. For any $\tau \preceq \sigma$ we denote $\tau^{\perp}=\left\{m \in M_{\mathbb{R}} \mid\langle m, n\rangle \geq 0\right.$ for all $\left.n \in \sigma\right\}$ and $\tau_{M}^{\perp}=\tau^{\perp} \cap M$; note that $\tau_{M}^{\perp}$ is the maximal subgroup of $\tau_{M}$. For $\tau \preceq \sigma$ we will denote $\tau^{\prime}$ for $\tau$ considered as maximal cone in its $\mathbb{R}$-linear span in $N_{\mathbb{R}}$; then $\tau_{M}^{\prime}$ equals $\sigma_{M} \cap \tau_{M}^{\perp}$. The associated monoid rings correspond to the orbit $\operatorname{orb}(\tau)=\operatorname{spec}\left(\mathbb{K}\left[\tau_{M}^{\perp}\right]\right)$ associated to $\tau$ and its closure $V(\tau)=\operatorname{spec}\left(\mathbb{K}\left[\tau_{M}^{\prime}\right]\right)$ in $U_{\sigma}$. Moreover, $U_{\tau}$ splits into a product $U_{\tau} \cong \operatorname{orb}(\tau) \times U_{\bar{\tau}}$, where $U_{\bar{\tau}}=\operatorname{spec}\left(\mathbb{K}\left[\bar{\tau}_{M}\right]\right)$ and $\bar{\tau}_{M}$ is the image of $\tau_{M}$ under the projection $M \rightarrow M / \tau_{M}^{\perp}$.

The notion of a $\sigma$-family is a simple reformulation of the notion of $M$-graded $\mathbb{K}\left[\sigma_{M}\right]$-modules which will help us to make the combinatorial content of such modules more explicit. The basic observation is that, given an $M$-graded $\mathbb{K}\left[\sigma_{M}\right]$-module

$$
E \cong \bigoplus_{m \in M} E_{m}
$$

its module structure is completely determined by the linear maps among the $E_{m}$ which are given by multiplication with monomials, i.e. for any $m \in \sigma_{M}$, by the $\mathbb{K}$-linear maps

$$
E_{m^{\prime}} \stackrel{\cdot \chi(m)}{\longrightarrow} E_{m+m^{\prime}}
$$

We define a relation on $M$ by setting $m \leq_{\sigma} m^{\prime}$ iff $m^{\prime}-m \in \sigma_{M}$. One checks immediately that $\leq_{\sigma}$ defines a preorder on $M$ with $m \leq_{\sigma} m^{\prime}$ and $m^{\prime} \leq_{\sigma} m$ iff $m-m^{\prime} \in \sigma_{M}^{\perp}$. We observe that $\chi\left(m^{\prime \prime}-m^{\prime}\right) \chi\left(m^{\prime}-m\right)=\chi\left(m^{\prime \prime}-m\right)$ whenever $m \leq_{\sigma} m^{\prime} \leq_{\sigma} m^{\prime \prime}$ and $\chi(m-m)=1$ for every $m \in M$. This way, we can consider every $M$-graded $\mathbb{K}\left[\sigma_{M}\right]$-module in a natural way as a representation of the preordered set $\left(M, \leq_{\sigma}\right)$.

Definition 2.6: A $\sigma$-family is a representation of the preordered set $\left(M, \leq_{\sigma}\right)$.

This definition of $\sigma$-family is equivalent to the definition given in [Per04], Definition 5.2. On the other hand, for every such representation which maps $m$ to some $\mathbb{K}$-vector space $E_{m}$, we can construct a $\mathbb{K}\left[\sigma_{M}\right]$-module $E=\bigoplus_{m \in M} E_{m}$. We have:

Proposition 2.7 ([Per04], Proposition 5.5): There is an equivalence of categories between the category $M$-graded $\mathbb{K}\left[\sigma_{M}\right]$-modules and the category of $\sigma$-families.

From now on we will not distinguish between an $M$-graded module and its induced representation of $\left(M, \leq_{\sigma}\right)$. By Proposition [2.2, the category of $\sigma$-families is equivalent to the category of representations of $M / \sigma_{M}^{\perp}$ with the induced partial order. By Proposition 2.2 we get an equivalence of categories between the category of $\sigma$-families and the category of $\sigma^{\prime}$-families. By Proposition 2.7 we get equivalently: 
Proposition 2.8: There is an equivalence of categories between the category of $M$-graded $\mathbb{K}\left[\sigma_{M}\right]$-modules and the category of $M / \sigma_{M}^{\perp}$-graded $\mathbb{K}\left[\sigma_{M}^{\prime}\right]$-modules.

Every $\mathbb{K}$-linear representation of $\left(M, \leq_{\sigma}\right)$ represents a directed system of $\mathbb{K}$-vector spaces. In Per04] $\S 5.4$, colimits of $\sigma$-families and their relations to the $\tau$-families for $\tau \preceq \sigma$ have been described. We add some observations which are direct consequences of the discussion in Per04] $\S 5.4$.

Definition 2.9: Let $E$ be an $M$-graded $\mathbb{K}\left[\sigma_{M}\right]$-module. Then we denote $\mathbf{E}$ the colimit of its associated $\sigma$-family.

Proposition 2.10: $\quad$ (i) Taking colimits is an exact functor from the category of $M$-graded $\mathbb{K}\left[\sigma_{M}\right]$-modules to the category of $\mathbb{K}$-vector spaces.

(ii) Let $E$ be an $M$-graded module and $\mathbf{E}$ its colimit. Then $\operatorname{dim}_{k} \mathbf{E}=\operatorname{rk} E$.

Proof. (ii) Just observe that the poset $\left(M, \leq_{\sigma}\right)$ is filtered and thus colimits are exact.

(iii) The rank of $E$ coincides with the rank of $E \otimes_{\mathbb{K}\left[\sigma_{M}\right]} \mathbb{K}[M]$ and the statement follows from the observation that $\mathbf{E} \cong\left(E \otimes_{\mathbb{K}\left[\sigma_{M}\right]} \mathbb{K}[M]\right)_{m}$ for any $m \in M$.

If $E$ is torsion-free, then every homomorphism $E_{m} \stackrel{\chi((m)}{\longrightarrow} E_{m+m^{\prime}}$ is injective and thus, by general properties of colimits, the canonical homomorphisms $E_{m} \longrightarrow \mathbf{E}$ are injective, too. This makes it possible to consider any torsion-free, $M$-graded $\mathbb{K}\left[\sigma_{M}\right]$-module as a family of subvector spaces of the limit vector space $\mathbf{E}$ together with some combinatorial information coming from the poset $\left(M, \leq_{\sigma}\right)$. As has been observed by Klyachko [Kly91 for the case of finitely generated torsion-free modules, this data can be organized in terms of multifiltrations (see also [Per04, $\S 5.5)$ of $\mathbf{E}$. In this work we will mostly be interested in the more special case, where $E$ is finitely generated and reflexive. The corresponding structural interpretation by Klyachko in terms of filtered vector spaces has been stated in Theorem 1.1. The morphisms in the category of filtered vector spaces are precisely those vector space homomorphisms which are compatible with the filtrations in the obvious sense. Given any family of filtrations $E^{k}(i)$ as in the Theorem 1.1, we can reconstruct the module $E$ by setting

$$
E_{m}=\bigcap_{k=1}^{n} E^{k}\left(l_{k}(m)\right) .
$$

For later use we state the following facts which are straightforward to check:

Proposition 2.11: Let $E$ be a finitely generated $M$-graded reflexive $\mathbb{K}\left[\sigma_{M}\right]$-module.

(i) $E$ splits into a direct sum of $M$-graded reflexive $\mathbb{K}\left[\sigma_{M}\right]$-modules $F \oplus G$ with filtrations $F^{k}(i)$ and $G^{k}(i)$ iff $\mathbf{E} \cong \mathbf{F} \oplus \mathbf{G}$ such that $E^{k}(i)=F^{k}(i) \oplus G^{k}(i)$ for every $k, i$.

(ii) Choose filtrations $F^{k}(i)$ of $\mathbf{E}$ such that $E^{k}(i) \subseteq F^{k}(i)$ for all $k$, $i$ and denote $F$ the associated reflexive $\mathbb{K}\left[\sigma_{M}\right]$-module. Then the identity on $\mathbf{E}$ induces an inclusion $E \hookrightarrow F$.

\subsection{Matlis duality, injective and projective modules}

We denote $M$ - $\mathbb{K}\left[\sigma_{M}\right]$-Mod the category of $M$-graded $\mathbb{K}\left[\sigma_{M}\right]$-modules and $M$ - $\mathbb{K}\left[\sigma_{M}\right]$-Mod $\operatorname{lits}_{f}$ abelian subcategory consisting of modules whose graded components are finite-dimensional. Following [GW78] $§ I I .1$ (see also [BH98] $§ 3.6$ and [MS04 $§ 11.3$ ), there exists a natural endofunctor of $M-\mathbb{K}\left[\sigma_{M}\right]$-Mod which is called the graded Matlis duality. Explicitly, an object $E$ in $M-\mathbb{K}\left[\sigma_{M}\right]$-Mod is mapped to

$$
\check{E}=\operatorname{Hom}_{\mathbb{K}}(E, \mathbb{K})=\bigoplus_{m \in M} \operatorname{Hom}_{\mathbb{K}}\left(E_{m}, \mathbb{K}\right)=\bigoplus_{m \in M} \operatorname{Hom}_{\mathbb{K}\left[\sigma_{M}\right]}^{M}(E(m), \mathbb{K}),
$$


such that the $M$-grading on $E$ is given by

$$
(\check{E})_{m}=\operatorname{Hom}_{\mathbb{K}}\left(E_{-m}, \mathbb{K}\right) .
$$

The module $\check{E}$ is called the graded Matlis dual of $E$. Its module structure over $\mathbb{K}\left[\sigma_{M}\right]$ for each monomial $\chi(m) \in \mathbb{K}\left[\sigma_{M}\right]$ in every degree $m^{\prime} \in M$ is given by

$$
\operatorname{Hom}_{\mathbb{K}}(\chi(m), \mathbb{K}): \operatorname{Hom}_{\mathbb{K}}\left(E_{-m^{\prime}}, \mathbb{K}\right) \longrightarrow \operatorname{Hom}_{\mathbb{K}}\left(E_{-m^{\prime}-m}, \mathbb{K}\right) .
$$

The graded Matlis duality functor is exact and satisfies similar properties as its non-graded counterpart, in particular it induces an anti-equivalence of categories between finitely generated $M$-graded $\mathbb{K}\left[\sigma_{M}\right]$-modules and artinian $M$-graded $\mathbb{K}\left[\sigma_{M}\right]$-modules (see [BH98], §3.6). More generally, if $E_{m}$ is finite-dimensional for every $m \in M$, then $\check{\check{E}} \cong E$ and Matlis duality induces an autoequivalence of $M-\mathbb{K}\left[\sigma_{M}\right]-\operatorname{Mod}_{f}$. In particular, we get:

$$
\operatorname{Hom}_{\mathbb{K}\left[\sigma_{M}\right]}^{M}(E, F)=\operatorname{Hom}_{\mathbb{K}\left[\sigma_{M}\right]}^{M}(\check{F}, \check{E})
$$

for any $E, F$ in $M-\mathbb{K}\left[\sigma_{M}\right]-\operatorname{Mod}_{f}$.

Now let $\tau \preceq \sigma$ be any face. Then $\tau_{M}=\sigma_{M}+\mathbb{Z} m_{\tau}$, where $m_{\tau}$ is an element in the relative interior of $\tau_{M}^{\prime}$. We see by the isomorphism $\mathbb{K}\left[\tau_{M}\right] \cong \mathbb{K}\left[\sigma_{M}\right]_{\chi\left(m_{\tau}\right)}$ that $\mathbb{K}\left[\tau_{M}\right]$ is a flat $\mathbb{K}\left[\sigma_{M}\right]$ module which is contained in $M-\mathbb{K}\left[\sigma_{M}\right]-\operatorname{Mod}_{f}$. In particular, we obtain a family $\mathbb{K}\left[\tau_{M}\right]_{\tau \prec \sigma}$ of flat $\mathbb{K}\left[\sigma_{M}\right]$-modules in $M-\mathbb{K}\left[\sigma_{M}\right]-\operatorname{Mod}_{f}$. On the other hand, in it was shown in [GW78] Theorem 1.3.3 that the indecomposable injective modules in $M$ - $\mathbb{K}\left[\sigma_{M}\right]$-Mod are of the form

$$
I\left(\mathbb{K}\left[\tau_{M}^{\prime}\right]\right)(m) \quad \text { for } \quad \tau \preceq \sigma \text { and } m \in M,
$$

where $I(E)$ denotes the graded injective hull for any module $E$ in $M-\mathbb{K}\left[\sigma_{M}\right]$-Mod. By Matlis duality, these injective modules can explicitly be described as

$$
I\left(\mathbb{K}\left[\tau_{M}^{\prime}\right]\right)(m)=\mathbb{K}\left[\tau_{M}\right]^{\sim}(m)
$$

(see [MS04], §11.4). By observing that $\operatorname{Hom}_{\mathbb{K}\left[\sigma_{M}\right]}^{M}\left(-, I\left(\mathbb{K}\left[\tau_{M}^{\prime}\right]\right)(m)\right)$ and $\operatorname{Hom}_{\mathbb{K}\left[\sigma_{M}\right]}^{M}\left(\mathbb{K}\left[\tau_{M}\right],-\right)$ restrict to endofunctors of $M-\mathbb{K}\left[\sigma_{M}\right]-\operatorname{Mod}_{f}$ and by the fact that Matlis duality is an autoequivalence of $M-\mathbb{K}\left[\sigma_{M}\right]-\operatorname{Mod}_{f}$, it is straightforward to show the following:

Proposition 2.12: The modules $\mathbb{K}\left[\tau_{M}\right](m)$ for $\tau \preceq \sigma$ and $m \in M$ form a complete set of irreducible projective objects in $M-\mathbb{K}\left[\sigma_{M}\right]-\operatorname{Mod}_{f}$.

In particular, the module $\mathbb{K}[M]$ is the unique indecomposable module which is injective as well as projective in $M-\mathbb{K}\left[\sigma_{M}\right]-\operatorname{Mod}_{f}$.

Remark 2.13: Note that Proposition 2.12 in general is not true for $M-\mathbb{K}\left[\sigma_{M}\right]$-Mod.

Remark 2.14: Note that the category $M-\mathbb{K}\left[\sigma_{M}\right]-\operatorname{Mod}_{f}$ is not a "good" category for the construction of injective or projective resolutions. Example 2.15 below shows that such resolutions must not necessarily exist in $M-\mathbb{K}\left[\sigma_{M}\right]-\operatorname{Mod}_{f}$. Later on we will restrict $M-\mathbb{K}\left[\sigma_{M}\right]-\operatorname{Mod}_{f}$ further in order to obtain a "good" category which contains finitely generated modules and their Matlis duals as well as the injective and projective modules discussed above.

Example 2.15: Consider the polynomial ring in one variable $\mathbb{K}[x]$ and the $\mathbb{Z}$-graded module $E=\bigoplus_{i \in \mathbb{Z}} \mathbb{K}(i)$, where $\mathbb{K}(i)$ denotes the simple module $\mathbb{K}$ shifted to degree $-i$. Then $E$ does not admit a nontrivial homomorphism from $\mathbb{K}\left[x, x^{-1}\right]$ and the first term of a minimal projective resolution of $E$ would necessarily be of the form

$$
\bigoplus_{i \in \mathbb{Z}} \mathbb{K}[x](i) \longrightarrow E \longrightarrow 0
$$

However, $\bigoplus_{i \in \mathbb{Z}} \mathbb{K}[x](i)$ is not contained in $\mathbb{Z}-\mathbb{K}[x]-\operatorname{Mod}_{f}$. 


\subsection{Minimizing projective and injective resolutions}

Recall that for a commutative local ring $R$ and any exact sequence $\cdots \rightarrow F_{i} \stackrel{\phi_{i}}{\rightarrow} F_{i-1}$ of free $R$-modules, the presence of a unit element in the matrix representing $\phi_{i}$ allows us to do row and column transforms in order to split of one free summand from $F_{i}$ and $F_{i-1}$, respectively. In our setting we consider a (generalized) projective resolution $P_{E}$ of $E$ in $M-\mathbb{K}\left[\sigma_{M}\right]-\operatorname{Mod}_{f}$, i.e. a complex of projective modules which is everywhere exact except at degree zero and $H_{0}\left(P_{E}\right) \cong E$. We assume that $P_{E, i}$ is a finite direct sum of projective modules for every $i \in \mathbb{Z}$. Then we can write the differentials $\phi_{i}: P_{E, i} \rightarrow P_{E, i-1}$ explicitly as

$$
\bigoplus_{k} \mathbb{K}\left[\tau_{i, k, M}\right]\left(m_{i, k}\right) \stackrel{\phi_{i}}{\longrightarrow} \bigoplus_{j} \mathbb{K}\left[\tau_{i-1, j, M}\right]\left(m_{i-1, j}\right),
$$

where $\phi_{i}$ can be represented by a monomial matrix $\phi_{i}=\left(\alpha_{i j k} \chi\left(m_{i j k}\right)\right)_{j k}$ with $\alpha_{i j k} \in \mathbb{K}$. We have $\alpha_{i j k}=0$ whenever $\tau_{i-1, j} \npreceq \tau_{i, k}$ or $m_{i-1, j} \mathbb{Z}_{\tau_{i, k}} m_{i, k}$. If $\alpha_{i j k} \neq 0$ then $\chi\left(m_{i j k}\right)$ is uniquely determined up to multiplication by some $\chi(m)$ with $m \in \tau_{i, k, M}^{\perp}$.

Lemma 2.16: With notation as above assume there are $i, j, k$ with aijk $\neq 0$ and $\tau_{i-1, j}=\tau_{i, k}$, $m_{i-1, j}-m_{i, k} \in \tau_{i}^{\perp}$. Then we can split off $\mathbb{K}\left[\tau_{i, j, M}\right]\left(m_{i, j}\right)$ and $\mathbb{K}\left[\tau_{i-1, j, M}\right]\left(m_{i-1, j}\right)$ from $P_{E, i}$ and $P_{E, i-1}$, respectively, i.e. there is a complex $P_{E}^{\prime}$, with $P_{E, i}^{\prime} \oplus \mathbb{K}\left[\tau_{i, j, M}\right]\left(m_{i, j}\right)=P_{E, i}$, and $P_{E, i-1}^{\prime} \oplus \mathbb{K}\left[\tau_{i-1, j, M}\right]\left(m_{i-1, j}\right)=P_{E, i-1}$, and $P_{E, l}^{\prime}=P_{E, l}$ otherwise, such that $P_{E}^{\prime}$ is a projective resolution of $E$.

Proof. If our conditions are fulfilled, the splittings $P_{E, i}^{\prime} \oplus \mathbb{K}\left[\tau_{i, k, M}\right]\left(m_{i, k}\right)=P_{E, i}$ and $P_{E, i-1}^{\prime} \oplus$ $\mathbb{K}\left[\tau_{i-1, j, M}\right]\left(m_{i-1, j}\right)=P_{E, i-1}$ split off for every degree $m_{i, k} \leq_{\tau_{i, k}} m$ a one-dimensional vector space from $P_{E, i}$ and $P_{E, i-1}$, respectively. So, the complex stays exact when we compose $\phi_{i+1}$ with the projection from $P_{E, i}$ to $P_{E, i}^{\prime}$, restrict $\phi_{i-1}$ to $P_{E, i-1}^{\prime}$, and replace $\phi_{i}$ by its restriction to $P_{E, i}$ composed with the projection from $P_{E, i-1}$ to $P_{E, i-1}^{\prime}$.

Note that in the proof of Lemma 2.16, rather than to refer to explicit row and column transformations, we have made use of the correspondence between $M$-graded $\mathbb{K}\left[\sigma_{M}\right]$-modules and representations of $\left(M, \leq_{\sigma}\right)$. Now we observe that Matlis duality maps $P_{E}$ to $\check{P}_{E}$, which is a (generalized) injective resolution of $\check{E}$, i.e. Matlis duality induces a correspondence in $M-\mathbb{K}\left[\sigma_{M}\right]-\operatorname{Mod}_{f}$ between projective resolutions of $E$ and injective resolutions of $\check{E}$ and vice versa. So, given any injective resolution $I_{E}$ of $E$ with differentials

$$
\bigoplus_{k} \mathbb{K}\left[\tau_{i, k, M}\right]^{\sim}\left(m_{i, k}\right) \stackrel{\phi_{i}}{\longrightarrow} \bigoplus_{j} \mathbb{K}\left[\tau_{i+1, j, M}\right]^{\sim}\left(m_{i+1, j}\right),
$$

we can represent $\phi_{i}$ by the transpose of the corresponding matrix $\check{\phi}_{-i}$ in $\check{I}_{E}$. Using this, we get the dual statement for injective resolutions.

Lemma 2.17: With notation as above and writing $\phi_{i}=\left(\alpha_{i j k} \chi\left(m_{i j k}\right)\right.$ assume there are $i, j, k$ with $\alpha_{i j k} \neq 0$ and $\tau_{i+1, j}=\tau_{i, k}, m_{i+1, j}-m_{i, k} \in \tau_{i}^{\perp}$. Then we can split off $\mathbb{K}\left[\tau_{i, k, M}\right]^{\sim}\left(m_{i, k}\right)$ and $\mathbb{K}\left[\tau_{i+1, j, M}\right]^{\sim}\left(m_{i+1, j}\right)$ from $I_{E, i}$ and $I_{E, i+1}$, respectively, i.e. there is a complex $I_{E}^{\prime}$, with $I_{E, i}^{\prime} \oplus$ $\mathbb{K}\left[\tau_{i, k, M}\right]^{\sim}\left(m_{i, k}\right)=I_{E, i}$, and $I_{E, i+1}^{\prime} \oplus \mathbb{K}\left[\tau_{i+1, j, M}\right]^{\sim}\left(m_{i+1, j}\right)=I_{E, i+1}$, and $I_{E, l}^{\prime}=I_{E, l}$ otherwise, such that $I_{E}^{\prime}$ is an injective resolution of $E$.

Definition 2.18: We call a projective (respectively injective) resolution minimal if we cannot split off summands as in Lemma 2.16 (respectively Lemma 2.17).

Having established minimality of projective and injective resolutions, we now recall the notions of graded Betti and Bass numbers. 
Definition 2.19: Let $E$ be a module in $M-\mathbb{K}\left[\sigma_{M}\right]-\operatorname{Mod}_{f}$ and $P_{E}=\cdots \rightarrow P_{E, 1} \rightarrow P_{E, 0}$, $I_{E}=I_{E}^{0} \rightarrow I_{E}^{1} \rightarrow \cdots$ minimal projective and injective resolutions of $E$, respectively. Then we have decompositions

$$
P_{E, i}=\bigoplus_{\tau \preceq \sigma} \bigoplus_{m \in M} \mathbb{K}\left[\tau_{M}\right](-m)^{\beta_{i}(\tau, m)} \quad \text { and } \quad I_{E}^{i}=\bigoplus_{\tau \preceq \sigma} \bigoplus_{m \in M} \mathbb{K}\left[\tau_{M}\right]^{\sim}(-m)^{b^{i}(\tau, m)} .
$$

We denote $\beta_{i}(\tau, \underline{c})$ the $i$-th graded Betti number of degree $\underline{c}$ with respect to $\tau$ and $b^{i}(\tau, \underline{c})$ the $i$-th graded Bass number of degree $\underline{c}$ with respect to $\tau$. For $\tau=\sigma$ we also write $\beta_{i}(m)$ and $b^{i}(m)$ instead of $\beta_{i}(\tau, m)$ and $b^{i}(\tau, m)$, respectively.

\subsection{Homogeneous coordinates and divisorial modules}

By a well-known construction due to Cox, every toric variety has a so-called homogeneous coordinate ring. In our situation we consider the polynomial ring $S=\mathbb{K}\left[x_{1}, \ldots, x_{n}\right]$ considered as monoid ring over $\mathbb{N}^{n}$ together with its natural $\mathbb{Z}^{n}$-grading. By the following exact sequence

$$
M \stackrel{L}{\longrightarrow} \mathbb{Z}^{n} \stackrel{\Phi}{\longrightarrow} A \longrightarrow 0
$$

where $L$ is defined by $L(m)=\left(l_{1}(m), \ldots, l_{n}(m)\right)$, we can endow $S$ with an $A$-grading by setting $\operatorname{deg}_{A} x^{\underline{c}}=\Phi(\underline{c})$ for every monomial $x \underline{c}$ in $S$. The image of $M$ in $\mathbb{Z}^{n}$ coincides with $M / \sigma_{M}^{\perp}$. In light of Proposition 2.8 we will assume without loss of generality that $L$ is injective. Then $\mathbb{K}\left[\sigma_{M}\right]$ (rather that $\mathbb{K}\left[\sigma_{M}^{\prime}\right]$ ) is realized as the degree zero subring of $S$ with respect to this grading. Geometrically, this can be interpreted as representation of $U_{\sigma}$ as a good quotient of the affine space $\mathbf{A}_{\mathbb{K}}^{n}$ by the diagonalizable group scheme spec $(\mathbb{K}[A])$. The irreducible torus invariant Weil divisors $D_{1}, \ldots, D_{n}$ on $U_{\sigma}$ are in one-to-one correspondence with $l_{1}, \ldots, l_{n}$ and we can identify $\mathbb{Z}^{n}$ with the free group generated over the $D_{i}$. Also we can identify $A$ with the Weil divisor class group $A_{d-1}\left(U_{\sigma}\right)$ and above sequence states that every Weil divisor class has a torus invariant representative which is determined up to a principal divisor associated to an element in $M$ which we interpret as a semi-invariant rational function on $U_{\sigma}$.

Any $\mathbb{Z}^{n}$-graded $S$-module $E$ can also be endowed with a natural $A$-grading by setting $E_{\alpha}:=$ $\bigoplus_{\underline{c} \in \Phi^{-1}(\alpha)} E_{\underline{c}}$ for every $\alpha \in A$. To better distinguish these gradings we introduce the following notation:

Definition 2.20: Let $E$ be a $\mathbb{Z}^{n}$-graded $S$-module. Then for any $\underline{c} \in \mathbb{Z}^{n}$ we denote its $A$-degree $\Phi(\underline{c})$ by

$$
E_{(\underline{c})}:=E_{\Phi(\underline{c})}=\bigoplus_{m \in M} E_{\underline{c}+L(m)} .
$$

So, every $\alpha \in A$ the $\mathbb{K}$-vector space $E_{\alpha}$ has a natural structure of a $\mathbb{K}\left[\sigma_{M}\right]$-module. In particular, taking degree zero is an exact functor

$$
{ }_{(0)}: \mathbb{Z}^{n}-S-\operatorname{Mod} \longrightarrow M-\mathbb{K}\left[\sigma_{M}\right] \text {-Mod. }
$$

A particular class of $\mathbb{K}\left[\sigma_{M}\right]$-modules arising this way are those coming from projective and injective modules in $\mathbb{Z}^{n}-S-\operatorname{Mod}_{f}$. For any $I \subset[n]$ we denote $x_{I}:=\prod_{i \in I} x_{i}$ and $S_{I}:=S_{x_{I}}$ the localization of $S$ at $x_{I}$. Then the projective and injective $S$-modules are given by $S_{I}(\underline{c})$ and $\check{S}_{I}(\underline{c})$ respectively (where degree-shifts are given by $\underline{c} \in \mathbb{Z}^{n}$ ). Explicitly, we have

$$
S_{I}(\underline{c})_{(0)}=\bigoplus_{m \in M_{I}^{\underline{c}}} \mathbb{K} \chi(m) \quad \text { and } \quad \check{S}_{I}(\underline{c})_{(0)}=\bigoplus_{-m \in M_{I}^{-} \underline{c}} \mathbb{K} \chi(m)
$$

where

$$
M_{I}^{\frac{d}{I}}=\left\{m \in M \mid l_{i}(m) \geq-d_{i} \text { for all } i \in I\right\}
$$

for any $\underline{d}=\left(d_{1}, \ldots, d_{n}\right) \in \mathbb{Z}^{n}$. 
Definition 2.21: Let $E$ be any module in $M-\mathbb{K}\left[\sigma_{M}\right]$-Mod. If $E$ is isomorphic to some $S_{I}(\underline{c})_{(0)}$, then we call $E$ divisorial. If $E$ is isomorphic to some, $\breve{S}_{I}(\underline{c})_{(0)}$ then we call $E$ codivisorial.

From our discussion of subsection 2.3 it follows that a divisorial module $S_{I}(\underline{c})_{(0)}$ is projective in $M-\mathbb{K}\left[\sigma_{M}\right]-\operatorname{Mod}_{f}$ iff $\left\{l_{i} \mid i \in I\right\}$ generate a face of $\sigma$. Analogously, a codivisorial module $\check{S}_{I}(\underline{c})_{(0)}$ is injective in $M-\mathbb{K}\left[\sigma_{M}\right]-\operatorname{Mod}_{f}$ iff $\left\{l_{i} \mid i \in I\right\}$ generate a face of $\sigma$. Hence, the classes of divisorial and codivisorial modules coincide with the classes of projective and injective modules, respectively, in $M-\mathbb{K}\left[\sigma_{M}\right]-\operatorname{Mod}_{f}$ iff $U_{\sigma}$ is smooth. If $U_{\sigma}$ is not smooth then the (co-)divisorial modules form a strictly larger class.

In the following lemma we collect some general properties of (co-)divisorial modules whose straightforward check we leave to the reader:

Lemma 2.22: Let $\underline{c}, \underline{c}^{\prime} \in \mathbb{Z}^{n}$ and $I, J \subseteq[n]$. Then:

$$
S_{J}\left(\underline{c}^{\prime}\right) \otimes_{S} \check{S}_{I}(\underline{c}) \cong \begin{cases}\check{S}_{I}\left(\underline{c}+\underline{c}^{\prime}\right) & \text { if } J \subseteq I \\ 0 & \text { else. }\end{cases}
$$

(ii)

$$
\operatorname{Hom}_{S}\left(S_{I}\left(\underline{c}^{\prime}\right), S_{J}(\underline{c})\right) \cong \operatorname{Hom}_{S}\left(\check{S}_{J}\left(\underline{c}^{\prime}\right), \check{S}_{I}(\underline{c})\right) \cong \begin{cases}S_{I}\left(\underline{c}-\underline{c}^{\prime}\right) & \text { if } I \subseteq J \\ 0 & \text { else. }\end{cases}
$$

(iii) $S_{I}(\underline{c})_{(0)}$ and $\check{S}_{I}(\underline{c})_{(0)}$ are indecomposable.

(iv) $S_{I}(\underline{c})_{(0)}$ is finitely generated as a $\mathbb{K}\left[\sigma_{M}\right]$-module iff $I=[n]$.

(v) Let $\tau \preceq \sigma$ and $m \in \sigma_{M}$ be in the relative interior of $\tau_{M}^{\prime}$, then the localization $\left(S_{I}(\underline{c})_{(0)}\right)_{\chi(m)}$ equals $S_{I \cup \tau(1)}(\underline{c})_{(0)}$.

(vi)

$$
\operatorname{Hom}_{\mathbb{K}\left[\sigma_{M}\right]}^{M}\left(S_{I}(\underline{c})_{(0)}, S_{J}\left(\underline{c}^{\prime}\right)_{(0)}\right)= \begin{cases}\mathbb{K} & \text { if } \underline{c}^{\prime} \leq \underline{c} \text { and } I \subseteq J \\ 0 & \text { else. }\end{cases}
$$

(vii) In particular, $S_{I}(\underline{c})_{(0)}$ is reflexive iff $I=[n]$.

(viii) Let $E$ be a nontrivial, torsion-free, and finitely generated $M$-graded $\mathbb{K}\left[\sigma_{M}\right]$-module. Then $\operatorname{Hom}_{\mathbb{K}\left[\sigma_{M}\right]}\left(S_{I}(\underline{c})_{(0)}, F\right)=0$ iff $I \neq[n]$.

Note that in particular property (表) implies that the sheaves over $U_{\sigma}$ associated to divisorial modules are quasi-coherent of rank one.

As remarked above, codivisorial modules are not injective in general. However, the following lemma shows that codivisorial modules are still be useful for computing local cohomology groups.

Proposition 2.23: Let $B \subseteq \mathbb{K}\left[\sigma_{M}\right]$ be a homogeneous ideal, $V \subset U_{\sigma}$ the corresponding $T$ invariant closed subscheme of $U_{\sigma}, \underline{c} \in \mathbb{Z}^{n}$ and $I \subseteq[n]$. Denote $\tau_{I}$ the minimal face of $\sigma$ such that $I \subseteq \tau_{I}(1)$.

(i) $\Gamma_{V} \check{S}_{I}(\underline{c})_{(0)}= \begin{cases}\check{S}_{I}(\underline{c})_{(0)} & \text { if the orbit corresponding to } \tau_{I} \text { is contained in } V . \\ 0 & \text { else. }\end{cases}$

(ii) The module $\check{S}_{I}(\underline{c})_{(0)}$ is $\Gamma_{V}$-acyclic.

(iii) Consider the preimage $\hat{V} \subset \mathbf{A}_{\mathbb{K}}^{n}$ of $V$ under the projection $\mathbf{A}_{\mathbb{K}}^{n} \rightarrow U_{\sigma}$. Then $\Gamma_{\hat{V}} \check{S}_{I}(\underline{c})=0$ iff $\Gamma_{V} \check{S}_{I}(\underline{c})_{(0)}=0$. 
Proof. For the case that $V$ is the minimal orbit in $U_{\sigma}$ statements (ii) and (iii) were shown in [TH86], Lemma 3.1. We leave the adaption of this proof to our slightly more general case to the reader.

Statement (iii) follows from (ii) and the observation that the orbit decomposition of $\hat{V}$ with respect to the toric structure of $\mathbf{A}_{\mathbb{K}}^{n}$ corresponds one-to-one to subsets $I$ of $[n]$ such that the orbit in $U_{\sigma}$ corresponding to $\tau_{I}$ is contained in $V$.

Later on we will use codivisorial resolutions in order to compute local cohomology with respect to to $V$ of general $M$-graded $\mathbb{K}\left[\sigma_{M}\right]$-Modules. As a first step, we will first determine the local cohomology of divisorial modules. Following GW78 $\S 3.2$, we can consider the Grothendieck-Cousin complex of any divisorial module $S_{I}(\underline{c})_{(0)}$. For this, we observe that we can decompose

$$
S_{I}(\underline{c}) \cong\left(\left(\bigotimes_{i \in I} \mathbb{K}\left[x_{i}, x_{i}^{-1}\right]\right) \otimes\left(\bigotimes_{i \in[n] \backslash I} \mathbb{K}\left[x_{i}\right]\left(c_{i}\right)\right)\right)
$$

where all tensor products are over $\mathbb{K}$, all $\mathbb{K}\left[x_{i}, x_{i}^{-1}\right], \mathbb{K}\left[x_{i}\right]$ are considered as $\mathbb{Z}$-graded, and $\underline{c}=\left(c_{1}, \ldots, c_{n}\right)$. Replacing the factors on the right hand side by the quasiisomorphic complexes $\bar{K}\left[x_{i}, x_{i}^{-1}\right] \rightarrow \mathbb{K}\left[x_{i}, x_{i}^{-1}\right] /\left(\mathbb{K}\left[x_{i}\right]\left(c_{i}\right)\right)$ for every $i \notin I$, we get an isomorphism of complexes

$$
S_{I}(\underline{c}) \cong\left(\left(\bigotimes_{i \in I} \mathbb{K}\left[x_{i}, x_{i}^{-1}\right]\right) \otimes\left(\bigotimes_{i \in[n] \backslash I} \mathbb{K}\left[x_{i}, x_{i}^{-1}\right] \rightarrow \mathbb{K}\left[x_{i}, x_{i}^{-1}\right] /\left(\mathbb{K}\left[x_{i}\right]\left(c_{i}\right)\right)\right)\right)=: C_{I, \underline{c}}^{\bullet}
$$

where now the right hand side denotes the total tensor product chain complex. Its degree-zero part $\left(C_{I, c}^{\bullet}\right)_{(0)}$ is a codivisorial — and by Proposition 2.23 therefore a $\Gamma_{V}$-acyclic - resolution of $S_{I}(\underline{c})_{(0)}$. Denote $\hat{\sigma}$ the combinatorial simplex on the set $[n]$ and for any $m \in M$ denote $\hat{\sigma}_{m}$ its full subsimplex supported on $i \in I$ with $l_{i}(m)<-c_{i}$. By inspection it turns out that the graded piece $\left(\left(C_{I, \underline{c}}^{\bullet}\right)_{(0)}\right)_{m}$ coincides with simplicial cochain complex of $\hat{\sigma}_{\underline{b}}$ over $\mathbb{K}$. Now, for any torus invariant closed subset $V \subseteq U_{\sigma}$, its complement is again a toric variety described by a subfan $\sigma_{V}$ of $\sigma$. We denote $\hat{\sigma}_{V, m}$ the simplicial subcomplex of $\hat{\sigma}_{m}$ such that $I \in \hat{\sigma}_{V, m}$ implies $\tau_{I} \preceq \sigma_{V}$ with the notation of Proposition 2.23. Now we consider the graded decomposition $H_{V}^{i}\left(S_{I}(\underline{c})_{(0)}\right) \cong \bigoplus_{m \in M} H_{V}^{i}\left(S_{I}(\underline{c})_{(0)}\right)_{m}$. Applying $\Gamma_{V}$ to $\left(C_{I, c}^{\bullet}\right)_{(0)}$ together with standard arguments involving the long exact cohomology sequence associated to $\hat{\sigma}_{m}$ and $\hat{\sigma}_{V, m}$ imply the following variation of a well-known standard result:

Proposition 2.24: For $I, \underline{c}$ as above and $i \geq 0$ we have for every $m \in M$

$$
H_{V}^{i}\left(S_{I}(\underline{c})_{(0)}\right)_{m} \cong H^{i-2}\left(\hat{\sigma}_{V, m} ; \mathbb{K}\right),
$$

where $H^{i-2}\left(\hat{\sigma}_{V, m} ; \mathbb{K}\right)$ denotes the $i-2$-th reduced cohomology of the simplicial complex $\hat{\sigma}_{V, m}$ with coefficients in $\mathbb{K}$.

\section{Resolutions}

In this section we will develop a general framework for divisorial and codivisorial resolutions of $M$-graded $\mathbb{K}\left[\sigma_{M}\right]$-modules. For this we will first consider $\mathbb{Z}^{n}$-graded modules over the polynomial ring $S=\mathbb{K}\left[x_{1}, \ldots, x_{n}\right]$ and introduce lcm- and gcd-lattices for such modules in subsection 3.1. In subsection 3.2 we identify the minimal admissible lcm-lattice of a reflexive module with the intersection poset of the vector space arrangement generated by its associated filtrations. In subsection 3.3 we introduce the category of combinatorially finite modules. Subsections 3.4 and 3.5 form the central parts of this section. In subsetion 3.4 we describe minimal projective resolutions of combinatorially finite modules in terms of their associated lcm-lattices. In 
subsection 3.5 we characterize these resolutions for reflexive modules as resolutions of vector space arrangements. In subsection 3.6 we give an interpretation of Miller's results on duality of projective and injective resolutions in terms of an autoequivalence of the derived category of combinatorially finite modules. Based on this, we discuss the computation of local cohomologies in subsection 3.7.

\section{1 lcm- and gcd-lattices}

Let $\underline{c}=\left(c_{1}, \ldots, c_{n}\right), \underline{c}^{\prime}=\left(c_{1}^{\prime}, \ldots, c_{n}^{\prime}\right) \in \mathbb{Z}^{n}$, then the least common multiple of $\underline{c}$ and $\underline{c}^{\prime}$ is defined as the exponent of the least common multiple of the monomials $x^{\underline{c}}$ and $x^{\underline{c}^{\prime}}$, i.e. $\operatorname{lcm}\left\{\underline{c}, \underline{c}^{\prime}\right\}=\left(\max \left\{c_{1}, c_{1}^{\prime}\right\}, \ldots, \max \left\{c_{n}, c_{n}^{\prime}\right\}\right)$. Analogously, the greatest common divisor is defined as $\operatorname{gcd}\left\{\underline{c}, \underline{c}^{\prime}\right\}=\left(\min \left\{c_{1}, c_{1}^{\prime}\right\}, \ldots, \min \left\{c_{n}, c_{n}^{\prime}\right\}\right)$. We set $\overline{\mathbb{Z}}:=\mathbb{Z} \cup\{-\infty, \infty\}$ which is totally ordered by $-\infty<n<\infty$ for all $n \in \mathbb{Z}$ and $-\infty$ denotes an actual sign change, i.e. $-\infty=-(\infty)$. We naturally extend the notions $\operatorname{lcm}\left\{\underline{c}, \underline{c}^{\prime}\right\}$ and $\operatorname{gcd}\left\{\underline{c}, \underline{c}^{\prime}\right\}$ to any $\underline{c}, \underline{c}^{\prime} \in \overline{\mathbb{Z}}^{n}$. Also, the partial order on $\mathbb{Z}^{n}$ extends naturally to a partial order on $\overline{\mathbb{Z}}^{n}$.

Any object $E$ in $\mathbb{Z}^{n}$-S-Mod can be extended to a representation of $\left(\overline{\mathbb{Z}}^{n}, \leq\right)$ by setting for every $\underline{n} \in \overline{\mathbb{Z}}^{n}$

$$
\bar{E}_{\underline{n}}:=\lim _{\leftarrow} E_{\underline{n}^{\prime}},
$$

where the limit is taken over all $\underline{n}^{\prime} \in \mathbb{Z}^{n}$ with $\underline{n} \leq \underline{n}^{\prime}$. We set $\bar{E}_{\underline{n}}$ to zero if this set is empty. This construction establishes a functor

$$
{ }^{-}: \mathbb{Z}^{n}-S \text {-mod } \longrightarrow \text { Representations of }\left(\overline{\mathbb{Z}}^{n}, \leq\right), \quad E \mapsto \bar{E} .
$$

Conversely, if we consider $\mathbb{Z}^{n}$ as a topological subspace of $\overline{\mathbb{Z}}^{n}$ and denote the inclusion $\iota: \mathbb{Z}^{n} \hookrightarrow$ $\overline{\mathbb{Z}}^{n}$, we obtain by restriction of sheaves the functor

$$
\iota^{-1}: \text { Representations of }\left(\overline{\mathbb{Z}}^{n}, \leq\right) \longrightarrow \mathbb{Z}^{n}-S \text {-mod, } \quad \bar{E} \mapsto \iota^{-1} \bar{E} .
$$

By the universal property of limits, the pair of functors ${ }^{-}$and $\iota^{-1}$ establishes an equivalence of categories between $\mathbb{Z}^{n}$-S-mod and its essential image in the category of representations of $\overline{\mathbb{Z}}^{n}$.

The partially ordered set $\overline{\mathbb{Z}}^{n}$ is a lattice with meet $\wedge$ and join $\vee$ being gcd and lcm, respectively. Any subset of $\overline{\mathbb{Z}}^{n}$ which is closed under joins (respectively meets) is called join sublattice (respectively meet sublattice). However in our context the following terms are more customary.

Definition 3.1: We call lcm-lattice any subset $\mathcal{L}$ of $\overline{\mathbb{Z}}^{n}$ which is closed under taking lcm and moreover for any element $\left(c_{1}, \ldots, c_{n}\right)$ in $\mathcal{L}$ we have $c_{i}<\infty$ for all $i$. We call gcd-lattice any subset $\mathcal{L}$ of $\overline{\mathbb{Z}}^{n}$ which is closed under taking gcd and moreover for any element $\left(c_{1}, \ldots, c_{n}\right)$ in $\mathcal{L}$ we have $c_{i}>-\infty$ for all $i$.

Let $\iota_{\mathcal{P}}: \mathcal{P} \hookrightarrow \overline{\mathbb{Z}}^{n}$ be any subposet considered as topological space by its subspace topology and $E$ in $\mathbb{Z}^{n}$-S-mod. Then the restriction of $\bar{E}$ to $\mathcal{P}$ is given by the sheaf theoretical pullback $\iota_{\mathcal{P}}^{-1} \bar{E}$. In terms of poset representations, this simply coincides with the restriction of $\bar{E}$ to $\mathcal{P}$. For the case that $\mathcal{P}$ is an lcm-lattice we define a functor which we think of as compression of the relevant information contained in $\bar{E}$ to a smaller - possibly finite - poset.

Definition 3.2: Let $\iota_{\mathcal{L}}: \mathcal{L} \hookrightarrow \overline{\mathbb{Z}}^{n}$ be an lcm-lattice. Then for any $E$ in $\mathbb{Z}^{n}$-S-mod we define

$$
\operatorname{zip}^{\mathcal{L}} E:=\operatorname{zip}_{\text {lcm }}^{\mathcal{L}} E:=\iota_{\mathcal{L}}^{-1} \bar{E} .
$$

Below we will introduce the Matlis dual notion zip gcd $\mathcal{G}_{\text {gcd }}$ but we will mostly be working with zip $\mathcal{L}_{\mathrm{lcm}}^{\mathcal{L}}$. Therefore we will usually drop the subscript "lcm" if there is no ambiguity.

Now, given a representation $F$ of an lcm-lattice $\mathcal{L}$, it is possible to extend this representation to a representation $F^{\prime}$ of $\overline{\mathbb{Z}}^{n}$ as follows. Given any $\underline{c} \in \overline{\mathbb{Z}}^{n}$, we have two possibilities: either there 
exists no element $\underline{c}^{\prime}$ in $\mathcal{L}$ with $\underline{c}^{\prime} \leq \underline{c}$, or there exists a unique maximal element $\max (\underline{c}) \in \mathcal{L}$ with $\max (\underline{c}) \leq \underline{c}$, which is the lcm of all $\underline{c}^{\prime} \in \mathcal{L}$ with $\underline{c}^{\prime} \leq \underline{c}$. In the first case we set $F_{\underline{c}}^{\prime}:=0$. In the second case we set $F_{\underline{c}}^{\prime}:=F_{\max (\underline{c})}$. For any $\underline{c} \leq \underline{c}^{\prime}$ such that $F_{\underline{c}}^{\prime} \neq 0$, we set as morphism $F^{\prime}\left(\underline{c}, \underline{c}^{\prime}\right):=F\left(\max (\underline{c}), \max \left(\underline{c}^{\prime}\right)\right)$ (and zero otherwise). This way we get a $\mathbb{K}$-linear representation of $\overline{\mathbb{Z}}^{n}$.

Definition 3.3: Let $\mathcal{L} \subseteq \overline{\mathbb{Z}}^{n}$ be an lcm-lattice, $F$ a representation of $\mathcal{L}$ and $F^{\prime}$ its extension to $\overline{\mathbb{Z}}^{n}$ as constructed above. Then we denote

$$
\text { unzip }^{\mathcal{L}} F:=\text { unzip }_{\text {lcm }}^{\mathcal{L}} F:=F^{\prime} .
$$

In general, applying the functor zip ${ }^{\mathcal{L}}$ to any module $E$ will destroy a lot of information about $E$ such that it is not possible to reconstruct $E$ from its restriction to $\mathcal{L}$.

Definition 3.4: Let $E$ be in $\mathbb{Z}^{n}-S$-mod and $\mathcal{L} \subseteq \overline{\mathbb{Z}}^{n}$ an lcm-lattice. Then we call $\mathcal{L}$ an $E$ admissible (or simply admissible) lcm-lattice if $E \cong$ unzip $^{\mathcal{L}} \circ$ zip $^{\mathcal{L}} E$.

Example 3.5: Let $I \subset[n]$ and $\underline{c} \in \mathbb{Z}^{n}$. Then the module $S_{I}(\underline{c})$ admits an admissible lcm-lattice which consists of only one element $p_{\underline{c}, I}=\left(p_{1}, \ldots, p_{n}\right) \in \overline{\mathbb{Z}}^{n}$, where

$$
p_{i}= \begin{cases}-\infty & \text { for } i \in I \\ c_{i} & \text { else. }\end{cases}
$$

The representation zip $\left\{p_{\underline{c}, I}\right\} S_{I}(\underline{c})$ then associates to $p_{\underline{c}, I}$ the vector space $\mathbb{K}$. Conversely, every element $p$ in $\overline{\mathbb{Z}}^{n}$ with $p_{i}<\infty$ for all $i$ together with the representation $\underline{p} \mapsto \mathbb{K}$ gives rise to a module $\bar{S}_{I}(\underline{c})$, where $I=\left\{i \in[n] \mid p_{i}=-\infty\right\}$ and $\underline{c} \in \mathbb{Z}^{n}$ any element such that $c_{i}=p_{i}$ for $i \notin I$.

Example 3.6: Let $S_{I}(\underline{c})$ be as in the previous example. Then for the Matlis duals $\check{S}_{I}(-\underline{c})$ we get a more complicated picture. A minimal admissible lcm-lattice $\mathcal{L}_{\underline{c}, I}$ is the lcm-lattice generated by $(-\infty, \ldots,-\infty)$ and $\left\{\underline{p}^{i}\right\}_{i \notin I}$ where

$$
p_{j}^{i}= \begin{cases}-c_{i}+1 & \text { for } j=i \\ -\infty & \text { else }\end{cases}
$$

The representation zip $\mathcal{L}_{\underline{\underline{c}}, I} \check{S}_{I}(-\underline{c})$ associates $\mathbb{K}$ to $(-\infty, \ldots,-\infty)$ and 0 to all other elements of $\mathcal{L}_{\underline{c}, I}$.

Example 3.7: Let $E$ in $\mathbb{Z}^{n}$-S-Mod be finitely generated and torsion free and denote $e_{1}, \ldots, e_{t}$ a minimal set of homogeneous generators of $E$ with degrees $\underline{c}_{1}, \ldots, \underline{c}_{t}$. Then the lcm-lattice generated by $\underline{c}_{1}, \ldots, \underline{c}_{t}$ is admissible for $E$. In case that $E$ is a monomial ideal, this lcm-lattice coincides with the classical lcm-lattice of GPW99]. For general torsion free modules it coincides with that of [CT03].

Example 3.8: Let $E=\mathbb{K}[x, y] /\left(x^{2}, x y, y^{2}\right)$. A minimal admissible lcm-lattice is generated by $\{0,(2,0),(1,1),(0,2)\}$ in $\mathbb{Z}^{2}$.

The proof of the following proposition yields a general construction of admissible posets:

Proposition 3.9: Any module in $\mathbb{Z}^{n}$-S-Mod admits an admissible poset.

Proof. Let $E$ be in $\mathbb{Z}^{n}-S$-Mod and $\bar{E}$ its extension to $\overline{\mathbb{Z}}^{n}$. Then for any $\underline{c} \in \mathbb{Z}^{n}$ we denote $I_{E}(\underline{c})$ the set of all those elements $\underline{c}^{\prime} \in \overline{\mathbb{Z}}^{n}$ which are minimal with the property that for all $\underline{c}^{\prime \prime} \in \overline{\mathbb{Z}}^{n}$ with $\underline{c}^{\prime} \leq \underline{c}^{\prime \prime} \leq \underline{c}$ the homomorphisms $\bar{E}_{\underline{c}^{\prime}} \rightarrow \bar{E}_{\underline{c}^{\prime \prime}}$ and $\bar{E}_{\underline{c}^{\prime \prime}} \rightarrow \bar{E}_{\underline{c}}$ are isomorphisms. Denote $\mathcal{L}_{E}$ 
the lcm-lattice generated by $\bigcup_{\underline{c} \in \mathbb{Z}^{n}} I_{E}(\underline{c})$. We claim that $\mathcal{L}_{E}$ is $E$-admissible. By construction we get that (unzip $\mathcal{L}_{E} \circ$ zip $\left.^{\mathcal{L}_{E}} E\right)_{\underline{c}} \cong E_{\underline{c}} \cong E_{\max (\underline{c})}$ for every $\underline{c} \in \mathbb{Z}^{n}$ (with notation as used in the context of Definition 3.3). We establish an isomorphism of representations by explicitly setting this isomorphism equal to the isomomorphism $E_{\max (\underline{c})} \rightarrow E_{\underline{c}}$. It is straightforward to check that this indeed induces an equivalence of representations.

Remark 3.10: Though the procedure in the proof of Proposition 3.9 always delivers an admissible lcm-lattice, this might not be an optimal (or minimal) choice in general. For the module of Example 3.8 we get that $\mathcal{L}_{E}$ is generated by $\{(-\infty,-\infty),(2,-\infty),(-\infty, 2), 0,(1,1)\}$ which is larger than the minimal lcm-lattice given in Example 3.8 ,

\section{2 lcm-lattices of reflexive modules}

The most important class of modules we want to understand are the finitely generated reflexive modules in $\mathbb{Z}^{n}-S$-Mod. Let $E$ be such a module, described by a family of full filtrations $\cdots \subseteq$ $E^{k}(i) \subseteq E^{k}(i+1) \subseteq \cdots \subseteq \mathbf{E}$ for every $k \in[n]$. Any such filtration is determined by its underlying flag of subvector spaces of $\mathbf{E}$ : for any $k$ denote $i_{1}^{k}<\cdots<i_{t_{k}}^{k}$ the maximal sequence of integers such that $\operatorname{dim} E^{k}\left(i_{j}^{k}-1\right)<\operatorname{dim} E^{k}\left(i_{j}^{k}\right)$ for any $1 \leq j \leq t_{k}$. Then the vector spaces $E^{k}\left(i_{1}^{k}\right) \subsetneq \cdots \subsetneq E^{k}\left(i_{t_{k}}^{k}\right)$ form a (partial) flag in $\mathbf{E}$. In particular, the set of subvector spaces $\left\{E^{k}\left(i_{j}^{k}\right) \mid k \in[n], 1 \leq j \leq t_{k}\right\}$ forms a subvector space arrangement in $\mathbf{E}$. We denote by $\mathcal{V}_{E}$ the subvector space arrangement in $\mathbf{E}$ which is generated by all intersections of the $E^{k}\left(i_{j}^{k}\right)$. $\mathcal{V}_{E}$ clearly forms a partially ordered set with respect to inclusion. To avoid cumbersome notation, we introduce the following convention.

Convention 3.11: Let $\mathcal{V}$ (or $\mathcal{V}_{E}, \mathcal{V}_{F_{i}}, \ldots$ ), denote a vector space arrangement, i.e. a family of subvector space of some fixed vector space which is closed under taking intersections. Then by abuse of notation, we use the same symbol $\mathcal{V}\left(\right.$ or $\left.\mathcal{V}_{E}, \mathcal{V}_{F_{i}}, \ldots\right)$ to denote its underlying poset.

With is convention, we identify the partially ordered set $\mathcal{V}_{E}$ with a subset of $\mathbb{Z}^{n}$ by the following map:

$$
\mathcal{V}_{E} \ni X \mapsto \underline{i}^{X}:=\left(i_{1}^{X}, \ldots, i_{n}^{X}\right) \in \mathbb{Z}^{n},
$$

where $i_{k}^{X}:=\min \left\{i_{j}^{k} \mid X \subseteq E^{k}\left(i_{j}^{k}\right)\right\}$. This map is order preserving and we can thus identify the poset underlying $\mathcal{V}_{E}$ with a subposet of $\mathbb{Z}^{n}$. We even get:

Proposition 3.12: Let $E$ be a finitely generated reflexive module in $\mathbb{Z}^{n}-S$-Mod. Then the set $\mathcal{V}_{E}$ as defined above is a minimal E-admissible lcm-lattice.

Proof. We first show that $\mathcal{V}_{E}$ is an lcm-lattice. Let $X, Y \in \mathcal{V}_{E}$, then $\operatorname{lcm}\left\{\underline{i}^{X}, \underline{i}^{Y}\right\}=\left(\max \left\{i_{1}^{X}\right.\right.$, $\left.\left.i_{1}^{Y}\right\}, \ldots, \max \left\{i_{n}^{X}, i_{n}^{Y}\right\}\right)=:\left(j_{1}, \ldots, j_{n}\right)$. Denote $Z:=\bigcap_{k=1}^{n} E^{k}\left(j_{k}\right)$. We claim that $j_{k}=i_{k}^{Z}$ for every $k$. Assume there exists some $k$ such that $i_{k}^{Z}<j_{k}$. Then either $i_{k}^{Z}<i_{k}^{X}$ or $i_{k}^{Z}<i_{k}^{Y}$. But then either $X \nsubseteq E^{k}\left(i_{k}^{Z}\right)$ or $Y \nsubseteq E^{k}\left(i_{k}^{Z}\right)$. But then either $X \nsubseteq Z$ or $Y \nsubseteq Z$ which is a contradiction, as $Z$ by construction contains both $X$ and $Y$. Therefore $\mathcal{V}_{E}$ is an lcm-lattice.

To check that $\mathcal{V}_{E}$ is admissible, it suffices to verify that (unzip $\mathcal{V}_{E} \circ$ zip $\left.\mathcal{V}_{E} E\right)_{\underline{c}}$ is isomorphic to $E_{\underline{c}}$ for every $\underline{c} \in \mathbb{Z}^{n}$. As $\mathcal{V}_{E}$ does not contain any element $\underline{c}$ such that $c_{k}<i_{1}^{k}$ for any $k$ and $E_{\underline{c}}=0$ for any such $\underline{c}$, it suffices to assume that $\underline{c} \geq\left(i_{1}^{1}, \ldots, i_{1}^{n}\right)$. Consider any graded component $E_{\underline{c}}$. We denote $i_{\underline{c}}^{k}:=\min \left\{i_{j}^{k} \mid E_{\underline{c}} \subseteq E^{k}\left(i_{j}^{k}\right)\right\}$ and $\underline{i}_{\underline{c}}=\left(i_{\underline{c}}^{1}, \ldots, i_{\underline{c}}^{n}\right)$. We observe that $E_{\underline{i_{c}}}$ is an element of $\mathcal{V}_{E}$ and $E_{\underline{i_{c}}} \cong E_{\underline{c}}$. Also, $\underline{i}_{\underline{c}}$ is the lcm of all $\underline{i}_{X}$ such that $X \subseteq E_{\underline{\underline{c}}}$.

For $E$ reflexive, among all possible choices for $E$-admissible lcm-lattices, the vector space arrangement $\mathcal{V}_{E}$ is a distinguished choice:

Definition 3.13: Let $E$ and $\mathcal{V}_{E}$ be as above, then we call $\mathcal{V}_{E}$ the canonical E-admissible lcm-lattice. 


\subsection{Combinatorially finite modules}

Now we want establish for any given finite lcm-lattice $\mathcal{L}$ an equivalence of categories between representations of $\mathcal{L}$ and $S$-modules for which $\mathcal{L}$ is admissible.

Proposition 3.14: Let $\mathcal{L}$ be a finite lcm-lattice in $\overline{\mathbb{Z}}^{n}$. Then the functors zip $\mathcal{L}$ and unzip $\mathcal{L}$ establish an equivalence of categories between the category of representations of $\mathcal{L}$ and the category of modules in $\mathbb{Z}^{n}$-S-Mod for which $\mathcal{L}$ is admissible.

Proof. By definition, we have $E \cong$ unzip $^{\mathcal{L}} \circ$ zip $^{\mathcal{L}} E$. Now let $F$ be any representation of $\mathcal{L}$. We have to show that $F_{\underline{c}}=\left(\operatorname{zip}^{\mathcal{L}} \circ \text { unzip }^{\mathcal{L}} F\right)_{\underline{c}}$ for every $\underline{c} \in \mathcal{L}$. This is immediately clear if $\underline{c} \in \mathbb{Z}^{n}$. In the case that $\underline{c} \in \overline{\mathbb{Z}}^{n} \backslash \mathbb{Z}^{n}$, we have $\left(\operatorname{zip}^{\mathcal{L}} \text { o unzip } \mathcal{L}^{\mathcal{L}} F\right)_{\underline{c}} \cong \lim \left(\text { unzip }^{\mathcal{L}}\right)_{\underline{c}^{\prime}}$, where the limit runs over all $\underline{c}^{\prime} \geq \underline{c}$. By the finiteness of $\mathcal{L}$, there is a nonempty region in $\overline{\mathbb{Z}}^{n}$ of which $\underline{c}$ is the unique minimal element and $\left(\operatorname{unzip}^{-} \mathcal{L} F\right)_{\underline{c}^{\prime}}=F_{\underline{c}}$ for all $\underline{c}^{\prime}$ in this region. Therefore (unzip $\left.{ }^{\mathcal{L}} F\right)_{\underline{c}}$ and thus $\left(\text { zip }^{\mathcal{L}} \circ \text { unzip }^{\mathcal{L}} F\right)_{\underline{c}}$ must coincide with $\bar{F}_{\underline{\underline{c}}}$.

In the sequel we will only consider modules in $\mathbb{Z}^{n}$-S-Mod which admit a finite admissible lcm-lattice and whose graded components are finite-dimensional:

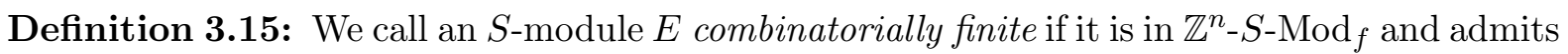
a finite $E$-admissible lcm-lattice $\mathcal{L} \subseteq \overline{\mathbb{Z}}^{n}$. We denote $\mathbb{Z}^{n}$-S-mod the category of combinatorially finite $S$-modules.

We have seen in Examples 3.5 and 3.6 that $\mathbb{Z}^{n}-S$-mod contains the injective and projective objects of $\mathbb{Z}^{n}-S-\operatorname{Mod}_{f}$. The following proposition whose proof we leave to the reader shows that $\mathbb{Z}^{n}-S$-mod moreover contains finitely generated modules and their Matlis duals:

Proposition 3.16: The category of combinatorially finite $S$-modules is abelian and closed under $\operatorname{Hom}_{S}, \otimes_{S}$, localization at monomials, and Matlis duality.

We can now introduce dual notion of associated gcd-lattices. Note that a subposet $\mathcal{G} \subseteq \overline{\mathbb{Z}}^{n}$ is a gcd-lattice iff $-\mathcal{G}$ is an lcm-lattice.

Definition 3.17: Let $E$ be an $S$-module in $\mathbb{Z}^{n}-S$-mod and $\mathcal{G}$ a finite gcd-lattice in $\overline{\mathbb{Z}}^{n}$. Then $\mathcal{G}$ is $E$-admissible if $-\mathcal{G}$ is an $\check{E}$-admissible lcm-lattice.

We can also define zip and unzip for gcd-lattices.

Definition 3.18: For any subposet $\mathcal{P}$ of $\overline{\mathbb{Z}}^{n}$ and any representation $F$ of $\mathcal{P}$, denote by $F^{\text {op }}$ the dual representation of the poset $-\mathcal{P}$, which maps $\underline{c}$ to $\operatorname{Hom}_{\mathbb{K}}\left(F_{\underline{c}}, \mathbb{K}\right)$. Then for any $E$ in $\mathbb{Z}^{n}$-S-mod and any gcd-lattice $\mathcal{G}$ we set

$$
\operatorname{zip}_{\mathrm{gcd}}^{\mathcal{G}} E:=\left(\operatorname{zip}_{\mathrm{lcm}}^{-\mathcal{G}} \check{E}\right)^{\text {op }}
$$

For a representation $F$ of $\mathcal{G}$ we set

$$
\text { unzip }_{\mathrm{gcd}}^{\mathcal{G}} F:=\left(\operatorname{unzip}_{\mathrm{lcm}}^{-\mathcal{G}} F^{\mathrm{op}}\right) \text {. }
$$

We leave it to the reader to formulate the gcd-version of Proposition 3.14.

Now we can introduce the notion of combinatorial finiteness for general rings $\mathbb{K}\left[\sigma_{M}\right]$ with homogeneous coordinate ring $S$ :

Definition 3.19: A module $E$ in $M-\mathbb{K}\left[\sigma_{M}\right]-\operatorname{Mod}_{f}$ is called combinatorially finite if there exists some $F \in \mathbb{Z}^{n}$-S-mod such that $E \cong F_{0}$. We denote $M$ - $\mathbb{K}\left[\sigma_{M}\right]$-mod the full category of $M-\mathbb{K}\left[\sigma_{M}\right]-\operatorname{Mod}_{f}$ of combinatorially finite modules. 
At this point we could, similar as in Proposition 3.16, try to proceed by investigating general properties of the category $M-\mathbb{K}\left[\sigma_{M}\right]$-mod. However, our approach instead will be to construct resolutions for a given $\mathbb{K}\left[\sigma_{M}\right]$-module $E$ by constructing a resolution for some appropriate $S$ module $F$ with $F_{0}=E$.

We conclude this subsection with the following observation:

Theorem 3.20: The category $M-\mathbb{K}\left[\sigma_{M}\right]-\bmod$ is a Krull-Schmidt category.

Proof. It suffices to show that for any combinatorially finite modules $E, F$ the $\mathbb{K}$-vector space $\operatorname{Hom}_{\mathbb{K}\left[\sigma_{M}\right]}^{M}(E, F)$ is finite-dimensional. For this, we choose combinatorially finite $S$-modules $E^{\prime}$ and $F^{\prime}$ with $E_{(0)}^{\prime}=E$ and $F_{(0)}^{\prime}=F$, respectively, and a finite lcm-lattice $\mathcal{L}$ which is admissible for both $E^{\prime}$ and $F^{\prime}$. Then $\operatorname{Hom}_{\mathbb{K}\left[\sigma_{M}\right]}^{M}(E, F)$ is a subvector space of $\bigoplus_{\underline{c} \in \mathcal{L}} \lim _{\longleftarrow} E_{m}$, where $\lim _{\longleftarrow} E_{m}$ runs over all $m \in M$ with $\underline{c} \leq m$ and hence is finite-dimensional.

\subsection{Using lcm- and gcd-lattices to compute resolutions}

In this subsection we will show that we can construct projective or injective resolutions for combinatorially finite $\mathbb{K}\left[\sigma_{M}\right]$-modules by computing the corresponding resolutions in an appropriate category of representations of admissible posets. The category of representations of a poset is equivalent to the category of modules over the incidence algebra of the associated Hasse diagram. We will present some basic facts about such representations and refer to [ASS06] for general overview of the theory of path algebras.

Let $\mathcal{P}$ be a finite poset. There is a bijection between the elements of $\mathcal{P}$ and the indecomposable projective object in the category of representations of $\mathcal{P}$, where for any $x \in \mathcal{P}$ its unique associated projective object $P_{x}$ is given by:

$$
P_{x, y}= \begin{cases}\mathbb{K} & \text { if } x \leq y \\ 0 & \text { else }\end{cases}
$$

together with identity homomorphisms $P_{x, y} \rightarrow P_{x, z}$, whenever $x \leq y \leq z$. Similarly, there is a bijection between $\mathcal{P}$ and the indecomposable injective objects. For $x \in \mathcal{P}$ its injective module $I_{x}$ is given by:

$$
I_{x, y}= \begin{cases}\mathbb{K} & \text { if } y \leq x \\ 0 & \text { else }\end{cases}
$$

together with identity homomorphisms $I_{x, y} \rightarrow I_{x, z}$, whenever $y \leq z \leq x$.

Every representation of $\mathcal{P}$ admits a finite projective as well as a finite injective resolution (see Proposition 3.21 below). As these resolutions will be crucial for our applications later on, we give now an explicit algorithmical description on how to obtain minimal projective resolutions. Let $F$ be any representation of $\mathcal{P}$. For any $x \in \mathcal{P}$, we can split the vector space $F_{x}$ as follows:

$$
F_{x} \cong F_{x, \leq} \oplus F_{x,>}
$$

where $F_{x, \leq}=\sum_{y<x} F(y, x)\left(F_{y}\right)$. By fixing a basis of $F_{x,>}$ we get an isomorphism $\mathbb{K}^{n_{x}^{0}} \cong F_{x,>}$ which gives rise to a natural homomorphism of $\mathcal{P}$-representations

$$
P_{x}^{n_{x}} \rightarrow F
$$

mapping the generators of $\left(P_{x}^{n_{x}^{0}}\right)_{x}$ to the basis of of $F_{x,>}$. Consequently, we get a short exact sequence of $\mathcal{P}$-representations

$$
0 \longrightarrow F^{1} \longrightarrow \bigoplus_{x \in \mathcal{P}} P_{x}^{n_{x}^{0}} \longrightarrow F \longrightarrow 0 .
$$


The kernel $F^{1}$ then is the 1st syzygy of $F$. By iterating, we obtain a projective resolution:

$$
0 \longrightarrow \bigoplus_{x \in \mathcal{P}} P_{x}^{n_{x}^{t}} \longrightarrow \cdots \longrightarrow \bigoplus_{x \in \mathcal{P}} P_{x}^{n_{x}^{0}} \longrightarrow F \longrightarrow 0
$$

This resolution is minimal by construction. Similarly, we can split $F_{x} \cong F^{x,<} \oplus F^{x, \geq}$, where $F^{x,<}=\bigcap_{x<y} \operatorname{ker} F(x, y)$ and $F^{x, \geq} \cong \mathbb{K}^{m_{x}^{0}}$ for $m_{x}^{0}=\operatorname{dim} F^{x, \geq}$. By iterating, we get a minimal injective resolution:

$$
0 \longrightarrow F \longrightarrow \bigoplus_{x \in \mathcal{P}} I_{x}^{m_{x}^{0}} \longrightarrow \cdots \longrightarrow \bigoplus_{x \in \mathcal{P}} I_{x}^{m_{x}^{r}} \longrightarrow 0
$$

From these constructions we can read off the following general property of $\mathcal{P}$-representations:

Proposition 3.21: Let $\mathcal{P}$ be a finite poset and $F$ a representation of $\mathcal{P}$. Then both projective and injective dimension of $\mathcal{P}$ are bounded by the maximal length of a chain in $\mathcal{P}$ minus one.

Note that by the general theory of path algebras it follows that projective and injective dimension coincide. Theorem 3.24 below will give even more effective bounds for posets which can be realized as lcm-lattices.

Proof. Let $x \in \mathcal{P}$ be minimal with the property that $F_{x} \neq 0$. Then, by above construction we get that $F_{x}^{1}=0$. So, if $x_{1}<x_{2} \cdots<x_{s}$ is a maximal chain in $\mathcal{P}$, then in the iteration we get for the $i$-th syzygy $F^{i}$ that $F_{x_{i}}^{i}=0$. This argument applies analogously to minimal injective resolutions.

Now let $E$ be any module in $\mathbb{Z}^{n}$-S-mod, and $\mathcal{L} \subset \overline{\mathbb{Z}}^{n}$ a finite $E$-admissible lcm-lattice. Then, as above, we can compute a minimal projective resolution

$$
0 \longrightarrow \bigoplus_{\underline{c} \in \mathcal{L}} P_{\underline{\underline{c}}}^{n_{\underline{c}}^{t}} \longrightarrow \cdots \longrightarrow \bigoplus_{\underline{c} \in \mathcal{L}} P_{\underline{\underline{c}}}^{n_{\underline{c}}^{0}} \longrightarrow \operatorname{zip}_{\mathrm{lcm}}^{\mathcal{L}} E \longrightarrow 0 .
$$

Unzipping yields an exact sequence

$$
0 \longrightarrow \bigoplus_{\underline{c} \in \mathcal{L}} \text { unzip }_{\text {lcm }}^{\mathcal{L}} P_{\underline{c}}^{n_{c}^{t}} \longrightarrow \cdots \longrightarrow \bigoplus_{\underline{c} \in \mathcal{L}} \text { unzip } \mathcal{L}_{\text {lcm }}^{\mathcal{L}} P_{\underline{c}}^{n_{c}^{0}} \longrightarrow E \longrightarrow 0 .
$$

Observing that unzip $\mathcal{L}_{\mathrm{lcm}}^{\mathcal{L}} P_{\underline{c}} \cong S_{I}\left(-\underline{c}^{\prime}\right)$ with $I=\left\{i \in[n] \mid c_{i}=-\infty\right\}$ and $\underline{c}^{\prime}=\left(c_{1}^{\prime}, \ldots, c_{n}^{\prime}\right) \in \mathbb{Z}^{n}$ such that $c_{i}^{\prime}=c_{i}$ whenever $i \notin[n]$ (see Example 3.5), we see that we have produced a minimal free resolution of $E$. Similarly, if $\mathcal{K}$ is a finite $E$-admissible gcd-lattice, we obtain an injective resolution of $E$ and a codivisorial resolution of $E_{0}$ :

$$
0 \longrightarrow E \longrightarrow \bigoplus_{\underline{c} \in \mathcal{L}} \operatorname{unzip}_{\mathrm{gcd}}^{\mathcal{K}} I_{\underline{c}}^{m_{\underline{c}}^{0}} \longrightarrow \cdots \longrightarrow \bigoplus_{\underline{c} \in \mathcal{L}} \operatorname{unzip}_{\mathrm{gcd}}^{\mathcal{K}} I_{\underline{c}}^{m_{\underline{c}}^{r}} \longrightarrow 0
$$

If we consider $S$ as homogeneous coordinate ring for $\mathbb{K}\left[\sigma_{M}\right]$, then the degree zero parts (unzip $\left.\mathcal{I}_{\text {lcm }}^{\mathcal{L}} P_{\underline{c}}^{n_{c}^{i}}\right)_{(0)}$ and $\left(\text { unzip }_{\text {gcd }}^{\mathcal{K}} I_{\underline{c}}^{m_{c}^{0}}\right)_{(0)}$ are divisorial, respectively codivisorial $\mathbb{K}\left[\sigma_{M}\right]$-modules. By the exactness of taking degree zero we thus get:

Theorem 3.22: Let $F$ be a combinatorially finite $\mathbb{K}\left[\sigma_{M}\right]$-module and $E$ a combinatorially finite $S$-module with $E_{(0)}=F$. Then the degree zero parts of complexes (2) and (3) yield divisorial, respectively codivisorial resolutions of $F$ over $\mathbb{K}\left[\sigma_{M}\right]$.

A first application of these constructions is a combinatorial characterization of projective and injective dimension of a combinatorially finite $S$-module. 
Theorem 3.23: Let $E$ be a module in $\mathbb{Z}^{n}-S$-mod. Then both the projective and injective dimension of $E$ are bounded by the maximal chain in an E-admissible finite $1 \mathrm{~cm}$ - and gcdlattice, respectively.

Proof. Follows immediately from Proposition 3.21 .

The following observation may be of independent interest for the representation theory of incidence algebras:

Theorem 3.24: Let $\mathcal{P}$ be a finite poset. If $\mathcal{P}$ is isomorphic either to an $\mathrm{lcm}$-lattice or a gcdlattice in some $\mathbb{Z}^{n}$, then both the projective and the injective dimension of $\mathcal{P}$ are bounded by $n$.

Proof. Projective and injective dimensions of any representation $F$ of $\mathcal{P}$ coincides with the projective and injective dimensions of unzip $\mathcal{l c m}_{\mathrm{P}}^{\mathcal{P}} F$ and $\operatorname{unzip}_{\mathrm{gcd}}^{\mathcal{P}} F$, respectively, as $S$-modules. The projective dimension of $S$ equals its injective dimension. By the Hilbert syzygy theorem, the projective dimension of $S$ is $n$.

Remark 3.25: Note that for Theorem 3.24 it is not necessary to consider embeddings into $\overline{\mathbb{Z}}^{n}$. Because $\mathcal{P}$ is finite, then, if we can an embedding of $\mathcal{P}$ into $\overline{\mathbb{Z}}^{n}$, we can also find an embedding into $\mathbb{Z}^{n}$.

\subsection{Reflexive modules and resolutions of vector space arrangements}

Let $E$ be a reflexive module in $\mathbb{Z}^{n}$-S-mod with filtrations $E^{k}(i)$ and numbers $i_{1}^{k}<\cdots<i_{t_{k}}^{k}$ as used in Proposition 3.12, denoting the steps in the filtrations. The set of vector spaces $E_{\underline{c}}=\bigcap_{k \in[n]} E^{k}\left(c_{i}\right) \subseteq \mathbf{E}$ form a vector space arrangement $\mathcal{V}_{E}$ in E. By Convention 3.11, we denote $\mathcal{V}_{E}$ also the underlying canonical poset in $\mathbb{Z}^{n}$. The first step of the algorithm described in subsection 3.4 yields the following short exact sequence:

$$
0 \longrightarrow E^{1} \longrightarrow F \longrightarrow E \longrightarrow 0
$$

where $F \cong \bigoplus_{\underline{c} \in \mathcal{V}_{E}} S(-\underline{c})^{n_{\underline{c}}}$ is a free cover of $E$ and $E^{1}$ the first syzygy of $E$. By construction, $\mathcal{V}_{E}$ is also admissible for $E^{1}$ and $F$. On the other hand, $E^{1}$ and $F$ both are reflexive and therefore come with their own canonical admissible posets $\mathcal{V}_{E^{1}}$ and $\mathcal{V}_{F}$, respectively, corresponding to vector space arrangements in the limit vector spaces $\mathbf{E}^{\mathbf{1}}$ and $\mathbf{F}$. From the arguments of Proposition 3.21 we conclude that $\mathcal{V}_{E^{1}}$ is a proper subposet of $\mathcal{V}_{E}$. For $\mathcal{V}_{F}$ we get:

Proposition 3.26: With above notation the posets $\mathcal{V}_{F}$ and $\mathcal{V}_{E}$ coincide as subsets of $\mathbb{Z}^{n}$.

Proof. By construction, we have

$$
F_{\underline{c}}=\bigoplus_{\substack{\underline{c}^{\prime} \in \mathcal{V}_{E} \\ \underline{c^{\prime}} \leq \underline{c}}} S\left(-\underline{c}^{\prime}\right)_{\underline{\underline{c}}}^{n^{\underline{c}^{\prime}}}, \quad \text { and } \quad \operatorname{dim} F_{\underline{c}}=\sum_{\substack{\underline{c}^{\prime} \in \mathcal{V}_{E} \\ \underline{c^{\prime} \leq \underline{c}}}} n_{\underline{c}^{\prime}} .
$$

In particular, $F_{\underline{c}}=F_{\underline{c}^{\prime}}$, where $\underline{c}^{\prime}=\max \left\{\underline{c}^{\prime \prime} \in \mathcal{V}_{E} \mid \underline{c}^{\prime \prime} \leq \underline{c}\right\}$. Therefore, $\mathcal{V}_{F} \subseteq \mathcal{V}_{E}$. To show equality, it suffices to check that $F_{\underline{c}^{\prime}} \neq F_{\underline{c}}$ whenever $\underline{c}^{\prime}<\underline{c} \in \mathcal{V}_{E}$. As in the proof of Proposition 3.12. $\underline{c}^{\prime}<\underline{c}$ implies that there exists some $k \in[n]$ such that $E_{\underline{c}} \subseteq E^{k}\left(c_{k}\right)$ but $E_{\underline{c}} \nsubseteq E^{k}\left(c_{k}^{\prime}\right)$ and $E_{\underline{c}}^{\prime} \subseteq E^{k}\left(c_{k}^{\prime}\right)$, where $\underline{c}=\left(c_{1}, \ldots, c_{n}\right), \underline{c}^{\prime}=\left(c_{1}^{\prime}, \ldots, c_{n}^{\prime}\right)$. In particular, $E_{\underline{c}^{\prime}} \subsetneq E_{\underline{c}}$. But then there must exist at least one $\underline{c}^{\prime \prime} \leq \underline{c}$, but $\underline{c}^{\prime \prime} \not \leq \underline{c}^{\prime}$ such that $n_{\underline{c}^{\prime \prime}}>0$. Then $\operatorname{dim} F_{\underline{c}}>\operatorname{dim} F_{\underline{c}^{\prime}}$ and the claim follows. 
Another way of phrasing Proposition 3.26 is that we have constructed a surjection of the vector space arrangement $\mathcal{V}_{E}$ by a combinatorially equivalent coordinate space arrangement $\mathcal{V}_{F}$ such that any $F_{\underline{c}} \in \mathcal{V}_{F}$ maps surjectively onto $E_{\underline{c}} \in \mathcal{V}_{E}$. The vector space arrangement $\mathcal{V}_{E^{1}}$ then can be considered as a syzygy arrangement of $\mathcal{V}_{E}$. By iterating, our free resolution of $E$ then yields a resolution of $\mathcal{V}_{E}$ in terms of coordinate vector space arrangements:

$$
0 \longrightarrow \mathcal{V}_{F_{t}} \longrightarrow \cdots \longrightarrow \mathcal{V}_{F_{0}} \longrightarrow \mathcal{V}_{E} \longrightarrow 0
$$

where every $\mathcal{V}_{F_{i}}$ is a coordinate vector space arrangement which is combinatorially equivalent to the $i$-th syzygy arrangement $\mathcal{V}_{E_{i}}$. This is a remarkable observation which yields a new class of invariants of vector space arrangements which can be expressed in combinatorial terms, where the vector space dimension in the arrangements $\mathcal{V}_{F_{i}}$ play the role of Betti numbers. In section 4 we will determine these invariants for the case of central hyperplane arrangements. However, so far it is not clear whether these have any use in the study of general vector space arrangements. From this discussion and Proposition 3.26 we conclude:

Theorem 3.27: Let $E$ be a $\mathbb{Z}^{n}$-graded, finitely generated, reflexive $S$-module. Then the poset of nonzero graded Betti numbers is determined by the embedding of the poset given by the underlying vector space arrangement $\mathcal{V}_{E}$ into $\mathbb{Z}^{n}$ For given $X \in \mathcal{V}_{E}$, the corresponding Betti number depends only on $\mathcal{V}_{E}$.

By the following construction our correspondence between reflexive $S$-modules and vector space arrangements at least provides a method to efficiently compute resolutions of arrangements (see Remark 3.31).

Definition 3.28: Let $\mathcal{V}$ be a finite vector space arrangement in some finite-dimensional vector space $V$. We say that a reflexive module $E$ in $\mathbb{Z}^{n}-S$-mod for some $n$ is a reflexive model for $\mathcal{V}$, if there exists an isomorphism of vector spaces $\mathbf{E} \rightarrow V$ which induces an isomorphism of vector space arrangements $\mathcal{V}_{E} \cong \mathcal{V}$.

Proposition 3.29: Every finite vector space arrangement has a reflexive model.

Proof. Let $\mathcal{V}$ be a finite vector space arrangements in some vector space $V$. We can assume without loss of generality that $\mathcal{V}$ is nontrivial and enumerate its elements which are different from $V$ and 0 by $X_{1}, \ldots, X_{n}$. Then we define a set of filtrations $V^{k}(i)$ of $V$ with

$$
V^{k}(i)= \begin{cases}0 & \text { for } i<0 \\ X_{k} & \text { for } i=0 \\ V & \text { for } i>0\end{cases}
$$

By Theorem 1.1 this data gives rise to a reflexive module in $\mathbb{Z}^{n}$-S-mod. Its underlying vector space arrangement by construction coincides with $\mathcal{V}$.

Of course, the choice of a reflexive model as in the proof of Proposition 3.29 is not canonical and far from being unique. For instance, for a given arrangement one could instead choose a minimal collection of flags whose intersection poset generate $\mathcal{V}$.

Remark 3.30: Note that our resolutions only depend on the actual arrangement. If, say, some vector space is contained in more than one filtration $E^{k}(i)$ associated to some reflexive module $E$, the resolution of the underlying vector space arrangement by coordinate arrangements does not depend on this multiplicity. However, the actual embedding of $\mathcal{V}_{E}$ in $\mathbb{Z}^{n}$ (and therefore the degrees which show up in the minimal resolution) does depend on possible multiplicities (see Example 3.32 below). 
Remark 3.31: Let a vector space arrangement $\mathcal{V}$ and a reflexive model $E_{\mathcal{V}}$ be given. By Proposition 2.11 (iii) we can choose an embedding of $E_{\mathcal{V}}$ into some free module $S(-\underline{c})^{\text {rk } E_{\mathcal{V}}}$ where $E^{k}\left(c_{k}\right)=0$ for every $k \in[n]$. If we are able to determine the first step of the resolution as in sequence (4), then we obtain a representation of $E_{\mathcal{V}}$ as the image of a monomial matrix $F_{0} \rightarrow S(-\underline{c})^{\mathrm{rk} E_{\mathcal{V}}}$. Then by computing a resolution of $E_{\mathcal{V}}$, say with help of a computer algebra system, we obtain also a resolution $\mathcal{V}$.

Example 3.32: Let $\mathcal{V}$ be a vector space arrangement in $V \cong \mathbb{K}^{r}$ generated by a family of subvector spaces $\left\{V_{1}, \ldots, V_{t}\right\}$ which has only trivial intersections, i.e. $V_{i} \cap V_{j}=\{0\}$ whenever $i \neq j$. We assume that the $V_{i}$ span $V$. Denote $I_{1} \sqcup \cdots \sqcup I_{t}=[n]$ some decomposition. If $E$ is given by filtrations

$$
V^{k}(i)= \begin{cases}0 & \text { for } i<i_{1}^{k} \\ V_{l} & \text { for } i_{1}^{k} \leq i<i_{2}^{k} \text { and } k \in I_{l} \\ V & \text { for } i_{2}^{k} \leq i\end{cases}
$$

and integers $i_{1}^{k}<i_{2}^{k}$ and $k \in[n]$, then we get as a minimal free resolution:

$$
0 \longrightarrow S\left(-\underline{i}_{2}\right)^{\sum_{l} \operatorname{dim} V_{l}-r} \longrightarrow \bigoplus_{l=1}^{t} S\left(-\underline{c}_{l}\right)^{\operatorname{dim} V_{l}} \longrightarrow E \longrightarrow 0 .
$$

Here, $\underline{i}_{2}=\left(i_{2}^{1}, \ldots, i_{2}^{n}\right)$ and $\underline{c}_{l}=\left(c_{l}^{1}, \ldots, c_{l}^{n}\right)$ with $c_{l}^{k}=i_{1}^{k}$ if $k \in I_{l}$ and $c_{l}^{k}=i_{2}^{k}$ else.

Example 3.33 (see also Bre08, $\S 6 \& \S 7$ ): ] Let $I \subset S$ be a monomial ideal and denote $T \subseteq \mathbb{Z}^{n}$ the minimal set of generators of its lcm-lattice in the sense of Example 3.7. The first term of a minimal resolution of $I$ is given by

$$
0 \longrightarrow I^{1} \longrightarrow \bigoplus_{\underline{c} \in T} S(-\underline{c}) \longrightarrow I \longrightarrow 0
$$

such that the first syzygy $I^{1}$ is a reflexive $S$-module. Consider the associated short exact sequence of limit vector spaces $0 \rightarrow \mathbf{I}^{\mathbf{1}} \rightarrow \mathbf{F} \rightarrow \mathbf{I} \rightarrow 0$, where $\operatorname{dim} \mathbf{I}=1$ and $\mathbf{F} \cong \bigoplus_{\underline{c} \in T} \mathbf{F}_{\underline{c}}$ with $\operatorname{dim} \mathbf{F}_{\underline{c}}=1$ for all $\underline{c} \in T$. The filtrations of $\mathbf{F}$ are given by

$$
F^{k}(i)=\bigoplus_{\underline{c} \in T, c_{k} \leq i} \mathbf{F}_{\underline{c}}
$$

The filtrations of $\mathbf{I}^{\mathbf{1}}$ then are given by the kernels of the homomorphisms $F^{k}(i) \rightarrow \mathbf{I}$. Betti numbers of monomial ideals are not purely combinatorial, as in general, for instance, they depend on the characteristic of $\mathbb{K}$. It would be of some interest to study this phenomenon in terms of vector space arrangements.

The approach presented here reduces the problem of constructing free resolutions to a straightforward linear algebra problem which allows to construct graded Betti numbers by iteratively analyzing linear dependencies. In general, we cannot expect to obtain closed forms for such resolutions apart from very easy cases, such as in example 3.32. However, as we will see in section 4, our approach is powerful enough to yield closed expressions for the case of hyperplane arrangements.

\subsection{Duality of resolutions and local cohomology}

We have seen so far that by using appropriate lcm- and gcd-lattices, projective and injective resolutions of combinatorially finite $S$-modules both can be constructed straightforwardly. In this subsection we will show that it actually suffices to consider only projective resolutions. 
More precisely, given some combinatorially finite $S$-module $E$, we will show that we from a given minimal projective resolution of $E$ a minimal injective resolution can directly be constructed.

For this consider the $\mathbb{Z}^{n}$-graded Grothendieck-Cousin complex of $S$, which provides a minimal injective resolution $0 \rightarrow S \rightarrow I_{S}$ in $\mathbb{Z}^{n}$-S-mod, with $I_{S}$ explicitly given by (see subsection 2.5):

$$
0 \longrightarrow \check{S}_{[n]}(\mathbf{1}) \longrightarrow \cdots \longrightarrow \bigoplus_{I \subseteq[n],|I|=n-i} \check{S}_{I}(\mathbf{1}) \longrightarrow \cdots \longrightarrow \check{S}(\mathbf{1}) \longrightarrow 0
$$

where $\mathbf{1}=(1, \ldots, 1) \in \mathbb{Z}^{n}$.

Now assume that we have a projective resolution of $E$, that is, a finite complex of projective modules $P_{E}$ in $\mathbb{Z}^{n}$-S-mod which is quasi-isomorphic to $E$, where $E$ is considered as a complex concentrated in degree 0 . Then we have the following chain of quasi-isomorphisms

$$
E \cong P_{E} \cong P_{E} \otimes_{S} S \cong P_{E} \otimes I_{S}
$$

where, due to Lemma 2.22 (ii), the last complex is a complex of injective modules in $\mathbb{Z}^{n}-S$-mod. This can be expressed more generally in the setting of derived categories as follows:

Lemma 3.34: Denote $K^{b}\left(\mathbb{Z}^{n}-S\right.$-proj) and $K^{b}\left(\mathbb{Z}^{n}-S\right.$-inj) the homotopy categories of bounded projective and injective complexes, respectively, in $\mathbb{Z}^{n}-S$-mod, and denote $D^{b}\left(\mathbb{Z}^{n}-S\right.$-mod) the bounded derived category of $\mathbb{Z}^{n}-S$-mod. Then the functors

$$
-\otimes_{S} I_{S}: K^{b}\left(\mathbb{Z}^{n}-S \text {-proj }\right) \rightarrow K^{b}\left(\mathbb{Z}^{n}-S \text {-inj }\right) \text { and } \operatorname{Hom}\left(I_{S},-\right): K^{b}\left(\mathbb{Z}^{n}-S \text {-inj }\right) \rightarrow K^{b}\left(\mathbb{Z}^{n} \text {-S -proj }\right)
$$

are well-defined and fit as mutually inverse functors into the following commutative diagram of equivalences of triangulated categories:

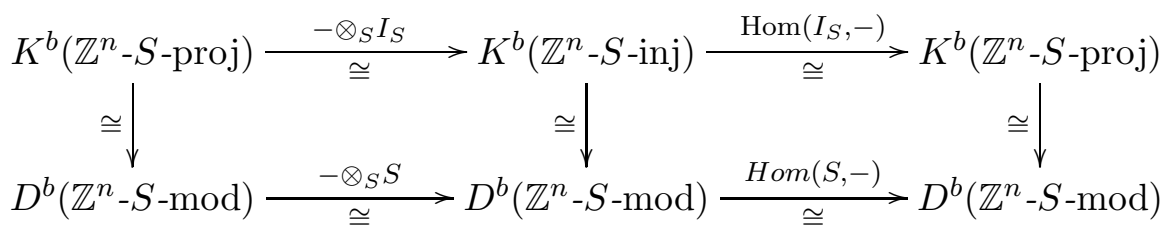

Proof. The vertical isomorphisms are the standard isomorphisms. It follows from Lemma 2.22 (ii) that the tensor product of a complex of projective modules yields a complex of injective modules. Likewise, it follows from 2.22 (iii) that $\operatorname{Hom}_{S}\left(I_{S},-\right)$ applied to a complex of injective modules yields a complex of projective modules. It is then clear that the horizontal arrows yield equivalences and that the diagram commutes.

For simplicity, we will assume from now that $E$ is a finitely generated module. Then a minimal projective resolution $P_{E}$ automatically is a free resolution. The complex $P_{E} \otimes_{S} I_{S}$ is not a resolution of $E$ in the traditional sense, as it is nonzero in degrees smaller than 0 . Our aim is to obtain from $P_{E} \otimes_{S} I_{S}$ a minimal injective resolution for $E$. To this end, consider the decomposition

$$
I_{S}=\bigoplus_{i=0}^{n} \bigoplus_{I \subseteq[n],|I|=n-i} \check{S}_{I}\left(e_{I}\right)[i-n] .
$$

Note that this decomposition is not a proper decomposition of $I_{S}$ into subcomplexes, i.e. we disregard the differential of $I_{S}$ for the moment. Then we can decompose

$$
P_{E} \otimes_{S} I_{S}=\bigoplus_{i=0}^{n} \bigoplus_{I \subseteq[n],|I|=n-i} P_{E} \otimes_{S} \check{S}_{I}(\mathbf{1})[i-n] .
$$


Explicitly, if we write $P_{E, i} \rightarrow P_{E, i-1}$ as $\bigoplus_{j} S\left(\underline{c}_{i, j}\right) \stackrel{\phi_{i}}{\rightarrow} \bigoplus_{k} S\left(\underline{c}_{i-1, j}\right)$, we get by Lemma 2.22 (ii) for $P_{E} \otimes \check{S}_{I}(\mathbf{1})$ :

$$
\bigoplus_{k} \check{S}_{I}\left(\underline{c}_{i, k}+\mathbf{1}\right) \longrightarrow \bigoplus_{j} \check{S}_{I}\left(\underline{c}_{i-1, j}+\mathbf{1}\right)
$$

Now, applying $\operatorname{Hom}_{S}\left(S_{I},-\right)$, we get by Lemma 2.22 (iii):

$$
\bigoplus_{k} S_{I}\left(\underline{c}_{i, k}+\mathbf{1}\right) \stackrel{\phi_{i}}{\longrightarrow} \bigoplus_{j} S_{I}\left(\underline{c}_{i-1, j}+\mathbf{1}\right) .
$$

That is, we can naturally identify the complex $\operatorname{Hom}_{S}\left(S_{I}, P_{E} \otimes_{S} \check{S}_{I}(\mathbf{1})\right)$ with the localization of the complex $P_{E}$ at the monomial $x^{I} \in S$. The complex $\left(P_{E}\right)_{x^{I}}$ then is a projective resolution of $E_{x^{I}}$ over $S_{x^{I}}$ which, in general, is no longer minimal. In the following theorem we will use this information and apply Lemma 2.17 to construct from $P_{E} \otimes_{S} I_{S}$ a minimal injective resolution for $E$.

Theorem 3.35: Let $E$ be a finitely generated $S$-module and $P_{E}$ a minimal free resolution of $E$. Then, by splitting off summands from $P_{E} \otimes_{S} I_{S}$, we obtain a minimal injective resolution $I_{E}$ of $E$. Its components with respect to the injectives $S_{I}$ are given by

$$
P_{E_{x I}} \otimes_{S} \check{S}_{I}(\mathbf{1})
$$

where $P_{E_{x^{I}}}$ denotes the minimal free resolution of $E_{x^{I}}$ over $S_{x^{I}}$.

Proof. First observe that $P_{E} \otimes_{S} \check{S}_{I}(\mathbf{1}) \cong\left(P_{E}\right)_{x^{I}} \otimes_{S} \check{S}_{I}(\mathbf{1})$ for every $I \subseteq[n]$. Consider the decomposition of $P_{E} \otimes_{S} I_{S}$ as above. Then, for every $I \subseteq[n]$, for every summand of the projective resolution $\left(P_{E}\right)_{x^{I}}$ which can be split of, we can can split of the corresponding summand of $P_{E} \otimes_{S} I_{S}$ by Lemmas 2.16 and 2.17. Therefore, reducing $\left(P_{E}\right)_{x^{I}}$ to a minimal resolution $P_{E_{x^{I}}}$ over $S_{x^{I}}$ for every $I \subseteq[n]$ simultaneously reduces $P_{E} \otimes_{S} I_{S}$ to a minimal injective resolution of $E$ with the stated properties.

Using Theorem 3.35, we can rederive a well-known correspondence between graded Betti and Bass numbers of finitely generated modules.

Corollary 3.36 (see [Mil00] $\S 5$ ): Let $E$ be a finitely-generated $\mathbb{Z}^{n}$-graded $S$-module and for $I \subset[n]$ denote $\tau_{I}$ the corresponding face of the positive orthant in $\mathbb{R}^{n}$. Then the graded Bass number $b^{i}\left(\tau_{I}, \underline{c}\right)$ equals the graded Betti number $\beta_{p_{I}-i}(\underline{c}+\mathbf{1})$ of the $S_{x^{I}}$-module $E_{x^{I}}$, where $p_{I}$ denotes the projective dimension of $E_{x^{I}}$ as an $S_{x^{I}}$-module.

Remark 3.37: As already mentioned, Corollary 3.36 is not a new result but has been proved in (essentially) the same generality in [Mil00. However, in loc. cit. the proof involves several technical ad-hoc methods, whereas by Lemma 3.34 this correspondence is just the specialization of a natural lift of the trivial autoequivalence of $D^{b}\left(\mathbb{Z}^{n}-S\right.$-mod) to the level of homotopy categories. Also, we can construct the full minimal injective resolution of a given module $E$, whereas in loc. cit., starting with the case of modules of finite length, an inductive argument for the pieces $P_{E} \otimes_{S} \breve{S}_{I}(\mathbf{1})[i-n]$ is given. Moreover, we can interpret now Alexander duality as studied in [Mil00] in more general terms by the autoequivalence of $D^{b}\left(\mathbb{Z}^{n}\right.$-S-mod) given by the Matlis duality functor.

Remark 3.38: The graded Grothendieck-Cousin complex is a very simplified version of the general Grothendieck-Cousin complex for local rings (see [Sha69]). However, the construction of Lemma 3.34 might be of more general interest, e.g. for studying Bass numbers and local cohomologies of modules over regular local rings. 
Another application is the following formula for computing the local cohomologies of modules in $\mathbb{Z}^{n}$-S-mod:

Theorem 3.39: Let $V \subseteq \mathbf{A}_{K}^{n}$ be a torus invariant closed subscheme and denote $R \Gamma_{V}$ : $D^{b}\left(\mathbb{Z}^{n}\right.$-S-mod $) \rightarrow D^{b}\left(\mathbb{Z}^{n}-S\right.$-mod) the right derived local cohomology functor. Then for any object $E$ in $D^{b}\left(\mathbb{Z}^{n}-S\right.$-mod) which has a projective representative $P_{E}$ in $K^{b}\left(\mathbb{Z}^{n}\right.$-S-proj) we get the following formula for local cohomology:

$$
H_{V}^{i}(E) \cong H^{i}\left(P_{E} \otimes_{S} R \Gamma_{V} S\right) .
$$

Proof. As in Lemma 3.34, we represent $E$ by a complex of injectives given by $P_{E} \otimes_{S} I_{S}$. Then clearly $R \Gamma_{V}(E) \cong \Gamma_{V}\left(P_{E} \otimes_{S} I_{S}\right)$. Then, decomposing $P_{E} \otimes_{S} I_{E}$ as before, we get by Lemma 2.23 (ii) that $\Gamma_{V}\left(P_{E} \otimes_{S} \check{S}_{I}(\mathbf{1})[i-n]\right)=0$ iff the support of $\check{S}_{I}\left(e_{I}\right)$ is not contained in $V$. Hence, the surviving part of $\Gamma_{V}\left(P_{E} \otimes_{S} \breve{S}_{I}(\mathbf{1})[i-n]\right)$ equals $P_{E} \otimes_{S} R \Gamma_{V} S$.

Remark 3.40: Theorem 3.39 is another variant of a well-known formula, e.g. see [BH98] Thm. 3.5.6 and [Mil00] Thm. 6.2.

\subsection{Computing local cohomologies}

Given a finitely generated module $E$ in $\mathbb{Z}^{n}-S$-mod, Theorem 3.35 tells us how to construct an admissible gcd-lattice. By Proposition 2.8, for every $I \subset[n]$, the category of $\mathbb{Z}^{n}$-graded $S_{x^{I}}$-modules is equivalent to the category of $\mathbb{Z}^{[n] \backslash I}$-graded $\left(S /\left(x_{i} \mid i \in I\right)\right)$-modules. Hence, for every $E_{x^{I}}$ we have an admissible lcm-lattice $\mathcal{L}_{I}$ in $\overline{\mathbb{Z}}^{[n] \backslash I}$. We embed these $\mathcal{L}_{I}$ into $\overline{\mathbb{Z}}^{n}$ by $\underline{c}=\left(c_{i} \mid i \in[n] \backslash I\right) \mapsto \iota_{I}(\underline{c})=\left(c_{1}^{\prime}, \ldots, c_{n}^{\prime}\right) \in \overline{\mathbb{Z}}^{n}$, where $c_{i}^{\prime}=\left\{\begin{array}{ll}\infty & \text { if } i \in I \\ c_{i}-1 & \text { otherwise. }\end{array}\right.$ Then by Theorem $3.35 \bigcup_{I \subset[n]} \iota_{I}\left(\mathcal{L}_{I}\right)$ is a superset of all degrees of objects contained in the minimal injective resolution of $E$. Hence, the gcd-lattice $\mathcal{G}$ generated by all $\iota_{I}\left(\mathcal{L}_{I}\right)$ in $\overline{\mathbb{Z}}^{n}$ is $E$-admissible in the sense of Definition 3.17 .

Now assume that we have constructed some minimal injective resolution $I_{E}$ and let $V \subseteq \mathbf{A}_{\mathbb{K}}^{n}$ be any closed torus invariant subscheme. Then $H_{V}^{i} E$ is determined completely once its graded parts $\left(H_{V}^{i} E\right)_{\underline{c}}=H^{i}\left(\Gamma_{V} I_{E}\right)_{\underline{c}}$ have been determined for every $\underline{c} \in \mathcal{G}$. For every $i \geq 0$ we decompose $\left(\bar{\Gamma}_{V} I_{E}\right)^{i}=: A^{i, \underline{c}} \oplus B^{i, \underline{c}}$ such that the first summand contains all summands $\check{S}_{I}(\underline{d})$ with $d_{i} \geq c_{i}$ for every $i \in[n] \backslash I$. Now, for any two summands $S_{J}(\underline{d})$ and $S_{I}\left(\underline{d}^{\prime}\right)$ of $\left(\Gamma_{V} I_{E}\right)^{i}$ and $\left(\Gamma_{V} I_{E}\right)^{i+1}$, respectively, we know by Lemma 2.22 (iii) that the corresponding entry of the representing matrix of the differential $\phi^{i}:\left(\Gamma_{V} I_{E}\right)^{i} \rightarrow\left(\Gamma_{V} I_{E}\right)^{i+1}$ (see Lemma 2.17) is zero unless $I \subseteq J$ and $d_{i} \leq d_{i}^{\prime}$ for every $i \in[n] \backslash J$. In particular, the image of restriction $\left.\phi^{i}\right|_{B^{i, \underline{c}}}$ is contained in $B^{i+1, \underline{c}}$. The following proposition will be useful.

Proposition 3.41: With above notation we have $\left(H_{V}^{i} E\right)_{\underline{c}} \cong\left(H^{i}\left(A^{\bullet}, \underline{c}\right)\right)_{\underline{c}} \cong\left(H^{i+1}\left(B^{\bullet, \underline{c}}\right)\right)_{\underline{c}}$ for every $\underline{c} \in \mathcal{G}$

Proof. By the above discussion the differential $\phi^{i}: A^{i} \oplus B^{i} \rightarrow A^{i+1} \oplus B^{i+1}$ decomposes into blocks as follows

$$
\phi^{i}=\left(\begin{array}{cc}
\phi_{A}^{i} & 0 \\
\phi_{A B}^{i} & \phi_{B}^{i}
\end{array}\right)
$$

with $\phi_{A}^{i}: A^{i} \rightarrow A^{i+1}, \phi_{A B}^{i}: A^{i} \rightarrow B^{i+1}$, and $\phi_{B}^{i}: B^{i} \rightarrow B^{i+1}$. Then we have complexes $\cdots \rightarrow A^{i} \stackrel{\phi_{A}^{i}}{\rightarrow} A^{i+1} \rightarrow \cdots$ and $\cdots \rightarrow B^{i} \stackrel{\phi_{B}^{i}}{\rightarrow} B^{i+1} \rightarrow \cdots$ which fit into a short exact sequence of complexes

$$
0 \longrightarrow B^{\bullet} \longrightarrow A^{\bullet} \oplus B^{\bullet} \longrightarrow A^{\bullet} \longrightarrow 0
$$

Then the assertion follows from the induced long cohomology sequence and the fact that the complex $A^{\bullet} \oplus B^{\bullet}$ is exact. 
Below we will mostly be interested in the local cohomologies $H_{x}^{i} E$ with respect to the torus fixed point $x$ in $\mathbf{A}_{\mathbb{K}}^{n}$ (or the maximal $\mathbb{Z}^{n}$-graded ideal of $S$, respectively). If a minimal free resolution $F_{\bullet} \rightarrow E \rightarrow 0$ is given, then by Theorem 3.39, $H_{x}^{i} E_{\mathcal{H}}$ coincides with the $i$-th cohomology of the complex $F_{\bullet} \otimes_{S} \check{S}(\mathbf{1})[-n]$. This implies that the "interesting" degrees are determined by the gcd-lattice $\mathcal{G}$ generated by the degrees of the nonzero graded Bass numbers $b^{i}(\underline{c})$ of $E$, i.e. by $\left\{\underline{c}-\mathbf{1} \mid \underline{c} \in \mathcal{L}_{\emptyset}\right\}$.

Definition 3.42: We call $\underline{c} \in \mathbb{Z}^{n}$ adjacent to $\underline{d} \in \mathcal{G}$ if $\underline{c} \leq \underline{d}$ and $\underline{c} \not \leq \underline{d}^{\prime}$ for all $\underline{d}^{\prime} \in \mathcal{G}$ with $\underline{d} \not \leq \underline{d}^{\prime}$.

For any $\underline{c} \in \mathbb{Z}^{n}$ we have $\left(H_{x}^{i} E\right)_{\underline{c}}=0$ if $\underline{c}$ is not adjacent to any $\underline{d} \in \mathcal{G}$ and $\left(H_{x}^{i} E\right)_{\underline{c}}=\left(H_{x}^{i} E\right)_{\underline{d}}$ if $\underline{c}$ is adjacent to $\underline{d}$.

Example 3.43: Consider vector space arrangements with reflexive model $E$ as in Example 3.32. The canonical admissible lcm-lattice is given by $\underline{i}_{2}$ and the $\underline{c}_{k}$. Then $\mathcal{G}$ is given by $\underline{d}_{P}:=\operatorname{gcd}\left\{\underline{c}_{l} \mid\right.$ $l \in P\}-1$ for every subset $P$ of $\{1, \ldots, t\}$. Explicitly, $\underline{d}_{P}=\left(d_{P}^{1}, \ldots, d_{P}^{n}\right)$, where $d_{P}^{k}=i_{1}^{k}-1$ if $k \in \bigcup_{p \in P} I_{p}$ and $d_{P}^{k}=i_{2}^{k}-1$ otherwise. The only possibly nontrivial local cohomologies are given by $\left(H_{x}^{n-1} E\right)_{\underline{c}}$ and $\left(H_{x}^{n} E\right)_{\underline{c}}$, where $\underline{c}$ is adjacent to some $\underline{d}_{p}$. Then $\operatorname{dim}\left(H_{x}^{n-1} E\right)_{\underline{d}_{P}}$ and $\operatorname{dim}\left(H_{x}^{n} E\right)_{\underline{d}_{P}}$ coincide with the dimension of the cohomologies of the complex

$$
0 \longrightarrow V^{1} \longrightarrow \bigoplus_{p \in P} V_{p} \longrightarrow 0
$$

which is concentrated in degrees $n-1$ and $n$, respectively. However, it is more convenient to read off the dimensions from the $n$-th and $(n+1)$-st cohomologies of the following complex which is concentrated in degrees $n$ and $n+1$ :

$$
0 \longrightarrow \bigoplus_{p \notin P} V_{p} \longrightarrow V \longrightarrow 0
$$

From this we read off

$$
\operatorname{dim}\left(H_{x}^{n-1} E\right)_{\underline{d}_{P}}=\sum_{i \notin P} \operatorname{dim} V_{i}-\operatorname{dim} \sum_{i \notin P} V_{i} \quad \text { and } \quad \operatorname{dim}\left(H_{x}^{n} E\right)_{\underline{d}_{P}}=\operatorname{dim} V-\operatorname{dim} \sum_{i \notin P} V_{i} .
$$

for every $P \subseteq\{1, \ldots, t\}$.

\section{Hyperplane arrangements}

In this section we want to apply our machinery to a special class of reflexive modules, whose associated filtrations form a hyperplane arrangement. These modules are introduced in subsection 4.1. In subsection 4.2 we introduce some notions from combinatorics and characterize some formulas in a form which is suitable for our applications. For a general reference for hyperplane arrangements and their associated combinatorics we refer to OT92. In subsection 4.3 construct free resolutions of hyperplane modules and determine their $\mathbb{Z}^{n}$-graded Betti numbers. In particular, we will show that these numbers are completely determined by the combinatorics of the associated hyperplane arrangement. Using these results and the duality of subsection 3.6. we will in subsection 4.4 completely determine the local cohomology of hyperplane modules with respect to the maximal $\mathbb{Z}^{n}$-graded ideal of $S$.

\subsection{Hyperplane modules}

A reflexive module $E$ in $\mathbb{Z}^{n}-S$-mod is called a hyperplane module if it is a reflexive model of a finite hyperplane arrangement in the sense of Definition 3.28 , Let $\mathcal{H}$ be a hyperplane 
arrangement in some vector space $V \cong \mathbb{K}^{r}$, generated by hyperplanes $H_{1}, \ldots, H_{t}$. For simplicity, we will assume that there are $\left\{k_{1}, \ldots, k_{t}\right\} \subseteq[n]$ such that the associated filtrations are of the form

$$
E^{k_{l}}(i)=\left\{\begin{array}{ll}
0 & \text { for } i<i_{k_{l}} \\
H_{l} & \text { for } i_{k_{l}} \leq i<j_{k_{l}} \\
V & \text { for } j_{k_{l}} \leq i,
\end{array} \quad \text { and } \quad E^{k}(i)=\left\{\begin{array}{ll}
0 & \text { for } i<j_{k} \\
V & \text { for } j_{k} \leq i .
\end{array} \text { for } k \notin\left\{k_{1}, \ldots, k_{t}\right\}\right.\right.
$$

for integers $i_{k_{j}}<j_{k_{j}}$ and $j_{k}$. In other words, every hyperplane is contained in precisely one filtration and we will allow for additional coordinates with trivial filtrations. The associated canonical admissible lcm-lattice $\mathcal{L}_{\mathcal{H}}$ in $\mathbb{Z}^{n}$ of $E_{\mathcal{H}}$ is given by $\mathcal{L}_{\mathcal{H}}=\left\{\underline{c}^{X}=\left(c_{1}^{X}, \ldots, c_{n}^{X}\right) \in \mathbb{Z}^{n} \mid\right.$ $X \in \mathcal{H}\}$, where

$$
c_{k}^{X}= \begin{cases}i_{k_{j}} & \text { if } k=k_{j} \text { for some } j \text { and } X \subseteq H_{j} \\ j_{k} & \text { else. }\end{cases}
$$

Recall that $\mathcal{H}$ is called essential if $\bigcap_{i} H_{i}=\{0\}$. If $\bigcap_{i} H_{i}=C$ for some nontrivial subvector space $C$ of $V$, we can apparently split $V \cong V^{\prime} \oplus C$ and $H_{i} \cong H_{i}^{\prime} \oplus C$, where $V^{\prime}:=V / C$ and $H_{i}^{\prime}:=H_{i} / C$ for every $i$. Obviously, the $H_{i}^{\prime}$ generate a hyperplane arrangement $\mathcal{H}^{\prime}$ in $V^{\prime}$. We observe that the isomorphism $V \cong V^{\prime} \oplus C$ induces an isomorphism of hyperplane arrangements between $\mathcal{H}$ and $\mathcal{H}^{\prime} \oplus C$. for $\mathcal{H}$ splits into a direct sum $E_{\mathcal{H}^{\prime}} \oplus S\left(-j_{1}, \ldots,-j_{n}\right)^{\operatorname{dim} C}$, where $E_{\mathcal{H}^{\prime}}$ is a reflexive model for the hyperplane arrangement $\mathcal{H}^{\prime}=\left\{H_{1}^{\prime}, \ldots, H_{t}^{\prime}\right\}$. So the minimal free resolutions for $E_{\mathcal{H}}$ and $E_{\mathcal{H}^{\prime}}$ coincide except for the 0-th term, where for $E_{\mathcal{H}}$ we get $S\left(-j_{1}, \ldots,-j_{n}\right)^{\operatorname{dim} C}$ as an extra summand. So below it will be safe to assume that $\mathcal{H}$ is essential.

\subsection{On the combinatorics of hyperplane arrangements}

We assume that $\mathcal{H}$ is an essential hyperplane arrangement. We consider $\mathcal{H}$ as a ranked poset with respect to the dimension.

Caution: Note that our this ordering on $\mathcal{H}$ is opposite to the standard ordering usually considered in the context of hyperplane arrangements see e.g. OT92. This might be somewhat confusing for some readers, but it is the more natural order with respect to our general setup.

For any subset $\mathcal{P}$ of $\mathcal{H}$, the Möbius function of $\mathcal{P}$ is defined as

$$
\mu^{\mathcal{P}}(X, Y)= \begin{cases}1 & \text { if } X=Y \\ -\sum_{X \leq Z<Y, \mathbb{Z} \in \mathcal{P}} \mu^{\mathcal{P}}(X, Z) & \text { if } X \leq Y \\ 0 & \text { else, }\end{cases}
$$

where we write $\mu$ for $\mu^{\mathcal{H}}$. Using the reduced dimension and the Möbius function, we take the following definitions from [GZ83].

Definition 4.1: Let $\mathcal{P}$ be any subset of $\mathcal{H}$.

(i) For $i \leq j \leq r$ the Whitney numbers are defined as

$$
w_{i j}^{\mathcal{P}}=\sum_{\substack{X \leq Y \in \mathcal{P} \\ \operatorname{dim} X=i, \operatorname{dim} Y=j}} \mu^{\mathcal{P}}(X, Y)
$$

We write $w_{i j}^{\mathcal{H}}=w_{i j}$ and $w_{i j}^{\{Y \leq X\}}=w_{i j}^{X}$ for some $X \in \mathcal{H}$.

(ii) The beta invariants are defined as

$$
\beta^{\mathcal{P}}=(-1)^{D} \sum_{d=0}^{D} d w_{d D}^{\mathcal{P}},
$$

where $D=\max \{\operatorname{dim} X \mid X \in \mathcal{P}\}$. 
(iii) For particular posets of the form $\mathcal{P}_{k, X}:=\{Y \leq X \mid \operatorname{dim} Y \leq k\} \cup\{X\}$ we write $\mu^{k, X}:=$ $\mu^{\mathcal{P}_{k, X}}, w_{i j}^{k, X}:=w_{i j}^{\mathcal{P}_{k, X}}, \beta^{k, X}:=\beta^{\mathcal{P}_{k, X}}$, and $\beta^{X}:=\beta^{\operatorname{dim} X-1, X}$.

For our constructions we introduce now another combinatorial characterization of beta invariants in terms of path length counting formulas.

Definition 4.2: Let $\mathcal{P}$ be any subset of $\mathcal{H}$ and denote $c$ any chain $X_{l}<X_{l-1}<\cdots<X_{0}=X$ in $\mathcal{P}$. Then we set $l(c):=l$, i.e. the length of $c$ minus one, and $\operatorname{dim} c:=\operatorname{dim} X_{l}$. For any $X \in \mathcal{H}$ we denote $C_{k, X}$ the set of chains in $\mathcal{P}_{k, X}$. For any $Y \in \mathcal{P}_{k, X}$ we denote $C_{k, Y, X} \subseteq C_{k, X}$ the subset of chains starting at $Y$.

With these notations we get the following lemma.

Lemma 4.3: Let $k \geq 0$ and $X \in \mathcal{H}$. Then

$$
\beta^{k, X}=(-1)^{k} \sum_{c \in C_{k, X}}(-1)^{l(c)} \operatorname{dim} c .
$$

Proof. We write

$$
\sum_{c \in C_{k, X}}(-1)^{l(c)} \operatorname{dim} c=\sum_{d=0}^{\operatorname{dim} X} d \sum_{Y \leq X, \operatorname{dim} Y=d} \sum_{c \in C_{k, Y, X}}(-1)^{l(c)} .
$$

Using the formula from [OT92, Prop. 2.37, we get:

$$
\begin{aligned}
(-1)^{k} \sum_{c \in C_{k}, X}(-1)^{l(c)} \operatorname{dim} c & =(-1)^{k} \sum_{d=0}^{\operatorname{dim} X} d \sum_{Y \in \mathcal{P}_{k, X}, \operatorname{dim} Y=d} \mu^{k, X}(Y, X) \\
& =(-1)^{k} \sum_{d=0}^{\operatorname{dim} X} d w_{d \operatorname{dim} X}^{k, X}=\beta^{k, X} .
\end{aligned}
$$

\subsection{Free resolutions}

Let $\mathcal{H}$ be a hyperplane arrangement. In this subsection we will a construct an explicit minimal resolution of $\mathcal{H}$ by coordinate arrangements $0 \rightarrow \mathcal{F}_{s} \stackrel{\phi_{s}}{\rightarrow} \ldots \stackrel{\phi_{7}}{\rightarrow} \mathcal{F}_{0} \stackrel{\phi_{0}}{\rightarrow} \mathcal{H} \rightarrow 0$. By results of subsection [3.4, for a hyperplane module $E_{\mathcal{H}}$ we get $\mathcal{F}_{i} \cong \bigoplus_{X \in \mathcal{H}} \mathbb{K}^{\beta_{i}\left(\underline{c}^{X}\right)}$, where $\beta_{i}\left(\underline{c}^{X}\right)$ denote the graded Betti numbers of $E_{\mathcal{H}}$. In particular, for $\beta_{i}(\underline{c})$ to be nonzero, $\underline{c}$ necessarily equals some $\underline{c}^{X}$ for $X \in \mathcal{H}$. By definition, for $i>0$, the $i$-th syzygy arrangement $\mathcal{H}^{i}$ consists of the arrangement of kernels of $\phi_{i-1}$ with the convention that $\mathcal{H}=\mathcal{H}^{0}$ is considered as the 0 -th syzygy. In particular, for any $i \geq 0$ we have a short exact sequence of vector space arrangements $0 \rightarrow \mathcal{H}^{i+1} \rightarrow \mathcal{F}_{i} \rightarrow \mathcal{H}^{i} \rightarrow 0$. As every $\mathcal{F}_{i}$ and every $\mathcal{H}^{i}$ can be considered as a $\mathbb{K}$-linear representation of the intersection poset of $\mathcal{H}$, we get for any $X \in \mathcal{H}$ a series of vector spaces $X=X^{0}, X^{1}, \ldots$ and $F_{0}^{X}, F_{1}^{X}, \ldots$ in $\mathcal{H}^{0}, \mathcal{H}^{1}, \ldots$ and $\mathcal{F}_{0}, \mathcal{F}_{1}, \ldots$, respectively. These fit into short exact sequences $0 \rightarrow X^{i+1} \rightarrow F_{i}^{X} \rightarrow X^{i} \rightarrow 0$ for any $i \geq 0$. We say that $X^{i}$ represents $X$ in the $i$-th syzygy.

Our construction will be done by induction on the number of generating hyperplanes. For this, we will assume that we already know how to construct such a resolution for $\mathcal{H}$ which is generated by $t$ hyperplanes $H_{1}, \ldots, H_{t}$. Now, if we add another hyperplane $H$, then the intersections $\{H \cap X \mid X \in \mathcal{H}\}$ induce a hyperplane arrangement $\mathcal{H}^{\prime}$ in $H$ which is generated by at most $t$ hyperplanes. So, our induction assumption applies to this arrangement as well. The induction step then will be to show that minimal resolutions of $\mathcal{H}$ and $\mathcal{H}^{\prime}$ can be constructed by splicing them together in a natural way. We will show the following theorem. 
Theorem 4.4: Let $\mathcal{H}$ be an essential hyperplane arrangement of rank $r$. Then there exists a minimal resolution $0 \rightarrow \mathcal{F}_{r-1} \rightarrow \cdots \rightarrow \mathcal{F}_{0} \rightarrow \mathcal{H} \rightarrow 0$ by coordinate arrangements with

$$
F_{i}^{Y}=\bigoplus_{\substack{X \leq Y \\ \operatorname{dim} X=i+1}} F_{i}^{X}
$$

for every $F_{i}^{Y} \in \mathcal{F}_{i}$. The differential is given by the sum of natural isomorphisms $F_{i}^{X} \cong X^{i}$ for any $X$ with $\operatorname{dim} X=i+1$. Moreover, for any $X \in \mathcal{H}$, we have $\operatorname{dim} X^{k}=\beta^{k, X}$ for any $X \in \mathcal{H}$ and any $k \geq 0$. In particular, we get $\operatorname{dim} X^{i}=\operatorname{dim} F_{i}^{X}=\beta^{X}$ for any $X \in \mathcal{H}$ with $\operatorname{dim} X=i+1$.

As an immediate corollary of Theorem 4.4 we obtain the following result for the graded Betti numbers of $E_{\mathcal{H}}$.

Theorem 4.5: Let $\mathcal{H}$ be an essential hyperplane arrangement and $E_{\mathcal{H}}$ a reflexive model. Then the graded Betti number $\beta_{i}\left(\underline{c}^{X}\right)$ of $E_{\mathcal{H}}$ is zero unless $\operatorname{dim} X=i+1$. If $\operatorname{dim} X=i+1$, then $\beta_{i}\left(\underline{c}^{X}\right)$ coincides with the beta invariant $\beta^{X}$ of $X$.

The rest of this subsection is devoted to the proof of Theorem 4.4. First, we introduce some more notation.

Definition 4.6: Let $\mathcal{H}$ be a hyperplane arrangement. For $k \geq 0$ we denote $\mathcal{H}_{k}=\{X \in \mathcal{H} \mid$ $\operatorname{dim} X=k\}$.

The following lemma states that the first step of the resolution can be verified directly.

Lemma 4.7: Set $\mathcal{F}_{0}:=\bigoplus_{X \in \mathcal{H}_{1}} X$ and $F_{0}^{Y}=\bigoplus_{Y \geq X \in \mathcal{H}_{1}} X$. Then the natural homomorphism of vector space arrangements $\mathcal{F}_{0} \stackrel{\phi_{0}}{\rightarrow} \mathcal{H}$ is surjective and minimal, i.e. $\phi_{0}$ does not factorize through another surjection $\mathcal{F}^{\prime} \rightarrow \mathcal{H}$, with $\mathcal{F}^{\prime}$ a coordinate arrangement of strictly lower dimension than $\mathcal{F}_{0}$.

Proof. The minimality follows from the fact that $\mathcal{H}$ is essential and $\mathcal{H}_{1}$ represents the set of its minimal nontrivial vector spaces. Therefore a coordinate vector space arrangement $\mathcal{F}^{\prime}$ surjecting onto $\mathcal{H}$ must at least have dimension $\left|\mathcal{H}_{1}\right|$. It follows from an easy induction on $\operatorname{dim} V$ and $t$ that the restriction of $\phi_{0}$ to $F_{0}^{X}$ is onto $X$ for every $X \in \mathcal{H}$.

We start now our induction. Because we only consider essential arrangements, we may begin with $t=r$ and by assuming without loss of generality that $H_{1}, \ldots, H_{r}$ are linearly independent and therefore generate an essential coordinate arrangement in $V$. It follows that $\phi_{0}$ from Lemma 4.7 is injective and therefore we get a minimal resolution of coordinate arrangements $0 \rightarrow \mathcal{F}_{0} \stackrel{\phi_{0}}{\rightarrow} \mathcal{H} \rightarrow 0$ for which the assertions of Theorem 4.4 trivially hold.

Now, for $t \geq r$ we have constructed by our induction assumption a minimal resolution of $\mathcal{H}$ and $\mathcal{H}^{\prime}$ by coordinate arrangements. Denote $\tilde{\mathcal{H}}$ the new hyperplane arrangement generated by $\mathcal{H}$ and $\mathcal{H}^{\prime}$. We want to combine the resolutions of $\mathcal{H}$ and $\mathcal{H}^{\prime}$ to a resolution of $\tilde{\mathcal{H}}$. We introduce some notation:

1. We denote $\cdots \mathcal{F}_{k} \stackrel{\phi_{k}}{\rightarrow} \mathcal{F}_{k-1}$ and $\cdots \mathcal{F}_{k}^{\prime} \stackrel{\phi_{k}^{\prime}}{\rightarrow} \mathcal{F}_{k-1}^{\prime}$ the resolutions of $\mathcal{H}$ and $\mathcal{H}^{\prime}$, respectively.

2. For every $k>0$ set $\mathcal{A}_{k}:=\mathcal{H}_{k}^{\prime} \backslash \mathcal{H}_{k}$.

3. For $X \in \mathcal{H}_{k}$ denote $X_{H}= \begin{cases}X \cap H \in \mathcal{A}_{k-1} & \text { if } X \nsubseteq H \\ 0 & \text { else. }\end{cases}$ 
In particular, we have $\mathcal{A}_{k-1}=\left\{X_{H} \mid X \in \mathcal{H}_{k}\right\}$. Our first step in the resolution then will be given by

$$
\tilde{\mathcal{F}}_{0}:=\mathcal{F}_{0} \oplus \bigoplus_{X \in \mathcal{A}_{1}} X \stackrel{\tilde{\phi}_{0}}{\longrightarrow} V
$$

where $\tilde{\phi}_{0}$ is the sum of $\phi_{0}$ and the restriction of $\phi_{0}^{\prime}$ to $\bigoplus_{X \in \mathcal{A}_{1}} X$. The free arrangement is given by $\tilde{F}_{0}^{Y}=\bigoplus_{X \leq Y, X \in \tilde{\mathcal{H}}_{1}} X$. The map $\tilde{\phi}_{0}$ is surjective by Lemma 4.7. We get $\operatorname{dim} \operatorname{ker} \tilde{\phi}_{0}=$ $\operatorname{dim} \operatorname{ker} \phi_{0}+\left|\mathcal{A}_{1}\right|$. Now consider for every $X \in \mathcal{H}_{1}$ the following commutative exact diagram:

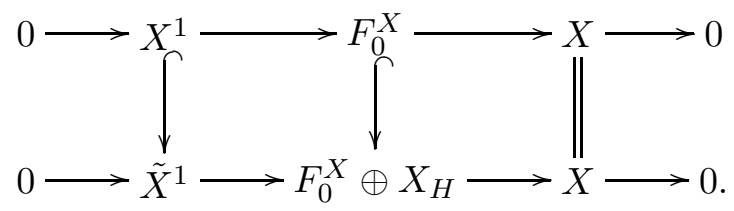

Denote $\psi_{1}$ the sum of all inclusions $\tilde{X}^{1} \hookrightarrow \tilde{F}_{0}$ with $X \in \mathcal{H}_{1}$, then we can read off from this diagram the next step in the resolution:

$$
\tilde{F}_{1}:=\bigoplus_{X \in \mathcal{H}_{2}} \tilde{X}^{1} \oplus \bigoplus_{X \in \mathcal{A}_{2}} X^{1} \stackrel{\tilde{\phi}_{1}}{\longrightarrow} \tilde{F}_{0}
$$

Here, $\tilde{\phi}_{1}$ is the sum of $\psi_{1}$ and of $\phi_{1}^{\prime}$ restricted to $\bigoplus_{X \in \mathcal{A}_{2}} X^{1}$. Now by diagram (5) and the observation that every $\tilde{X}^{1}$ with $X_{H} \neq 0$ projects nontrivially to $X_{H}$ and trivially to every other $Y_{H} \in \mathcal{A}_{1}$, we conclude that $\operatorname{rk} \psi_{1}=\operatorname{rk} \phi_{1}+\left|\mathcal{A}_{1}\right|=\operatorname{dim} \operatorname{ker} \tilde{\phi}_{0}$. It follows that $\operatorname{dim} \operatorname{ker} \tilde{\phi}_{1}=\operatorname{dim} \operatorname{ker} \phi_{1}+\sum_{X \in \mathcal{A}_{2}} \operatorname{dim} X^{1}$. To conclude, we observe that $\operatorname{dim} \operatorname{ker} \tilde{\phi}_{1}-\operatorname{dim} \operatorname{ker} \phi_{1}=$ $\sum_{X \in \mathcal{A}_{2}} \operatorname{dim} X^{1}$ and $\operatorname{rk} \tilde{\phi}_{1}-\operatorname{rk} \phi_{1}=\sum_{X \in \mathcal{A}_{1}} \operatorname{dim} X^{0}$.

Now we assume inductively that we have constructed $\tilde{\phi}_{i}: \mathcal{F}_{k} \rightarrow \mathcal{F}_{k-1}$ and that the inclusion $\mathcal{F}_{k} \hookrightarrow \bigoplus_{X \in \mathcal{H}_{k}} \tilde{X}^{k}$ induces an isomorphism ker $\psi_{k} \cong \operatorname{ker} \phi_{k}$, where $\psi_{k}$ denotes the restriction of $\tilde{\phi}$ to $\bigoplus_{X \in \mathcal{H}_{k+1}} \tilde{X}^{k}$. Moreover, assume that this isomorphism induces a natural isomorphism $\left.X^{k+1} \cong \operatorname{ker} \psi_{k}\right|_{\tilde{X}^{k}}$ for every $X \in \mathcal{H}_{k+2}$. Then we have the following commutative exact diagram for every $X \in \mathcal{H}_{k+2}$ :

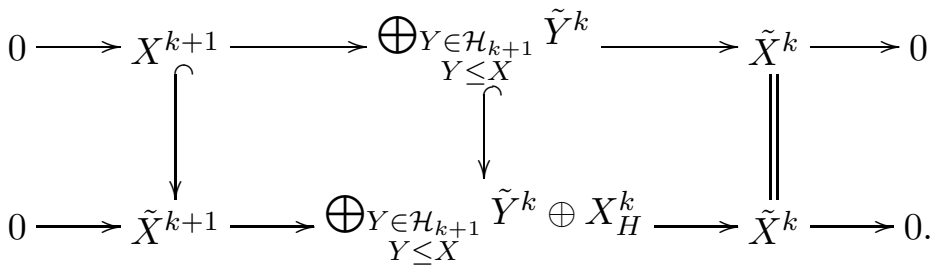

Denoting $\psi_{k+1}$ the sum of all inclusions $\tilde{X}^{k+1} \hookrightarrow \tilde{F}_{k}$ we set

$$
\tilde{F}_{k+1}:=\bigoplus_{X \in \mathcal{H}_{k+2}} \tilde{X}^{k+1} \oplus \bigoplus_{X \in \mathcal{A}_{k+1}} X^{k+1} \stackrel{\tilde{\phi}_{k+1}}{\longrightarrow} \tilde{F}_{k} .
$$

with $\tilde{\phi}_{k+1}$ the sum of $\psi_{k+1}$ and $\phi_{k+1}^{\prime}$ restricted to $\bigoplus_{X \in \mathcal{A}_{k+1}} X^{k}$. By observing that every $X \in \mathcal{H}_{k+1}$ with $X_{H} \neq 0$ the vector space $\tilde{X}^{k+1}$ projects nontrivially to $X_{H}^{k}$ and trivially to every other $Y_{H}^{k}$ with $Y_{H} \in \mathcal{A}_{k+1}$, we conclude that $\mathrm{rk} \psi_{k+1}=\operatorname{rk} \phi_{k+1}+\sum_{X \in \mathcal{A}_{k+1}}$. We conclude that $\operatorname{dim} \operatorname{ker} \tilde{\phi}_{k+1}-\operatorname{dim} \operatorname{ker} \phi_{k+1}=\sum_{X \in \mathcal{A}_{k+2}} \operatorname{dim} X^{k+1}$ and $\operatorname{rk} \tilde{\phi}_{k+1}-\operatorname{rk} \phi_{k+1}=\sum_{X \in \mathcal{A}_{k+1}} \operatorname{dim} X^{k}$. In particular, the image of $\tilde{\phi}_{k+1}$ coincides with the kernel of $\tilde{\phi}_{k}$ and by induction we obtain a minimal resolution of $\tilde{\mathcal{H}}$ by coordinate arrangements. This shows the first assertion of Theorem 4.4.

The following proposition proves the assertion on the dimensions of the vector spaces $X^{k}$. 
Proposition 4.8: $\operatorname{dim} X^{k}=\beta^{k, X}$ for any $X \in \mathcal{H}$. In particular, if $\operatorname{dim} X=i+1$, then $\operatorname{dim} X^{i}=\operatorname{dim} F_{i}^{X}=\beta^{X}$.

Proof. With the notation from Definition 4.2 we claim $\operatorname{dim} X^{k}=(-1)^{k} \sum_{c \in C_{k, X}}(-1)^{l(c)} \operatorname{dim} c$.

The claim is trivially true for $k=0$. Now we do induction on $k$ and assume that $k>0$. Then we have $\operatorname{dim} X^{k}=\operatorname{dim} F_{k-1}^{X}-\operatorname{dim} X^{k-1}$ and $\operatorname{dim} F_{k-1}^{X}=\sum_{Y \leq X, \operatorname{dim} Y=k} \operatorname{dim} Y^{k-1}$ by Theorem 4.4. Using our induction assumption, we get

$$
\begin{aligned}
\operatorname{dim} X^{k} & =\sum_{Y \in \mathcal{P}_{k, X}, \operatorname{dim} Y=k}(-1)^{k-1} \sum_{c \in C_{k-1, Y}}(-1)^{l(c)} \operatorname{dim} c-(-1)^{k-1} \sum_{c \in C_{k-1, X}}(-1)^{l(c)} \operatorname{dim} c \\
& =(-1)^{k} \sum_{c \in C_{k, X}}(-1)^{l(c)} \operatorname{dim} c .
\end{aligned}
$$

So the claim is proved and the proposition follows with Lemma 4.3 ,

Remark 4.9: In order to relate our results to earlier work on $\mathbb{Z}^{n}$-graded resolutions such as [CT03, [Tch07], we briefly explain how we can construct a matrix representation for a hyperperplane module. Assume that the $H_{i}$ are given by the orthogonal complement of linear forms $u_{1}, \ldots, u_{t}$ in $V^{*}$. Then we get a short exact sequence of $\mathbb{K}$-vector spaces

$$
0 \longrightarrow V \stackrel{\eta}{\longrightarrow} \mathbb{K}^{t} \stackrel{\xi}{\longrightarrow} \mathbb{K}^{t-r} \longrightarrow 0
$$

where $\eta$ is represented by a matrix whose $l$-th row is given by $u_{l}$ and $\xi$ is the corresponding cokernel map. It is not difficult to see that this sequence can be considered as associated to the following $\mathbb{Z}^{n}$-graded exact sequence:

$$
0 \longrightarrow E_{\mathcal{H}} \stackrel{\tilde{\eta}}{\longrightarrow} \bigoplus_{i=1}^{t} S\left(-\underline{c}^{H_{i}}\right) \stackrel{\tilde{\xi}}{\longrightarrow} S\left(-\underline{c}^{V}\right)^{t-r} .
$$

In particular, $\xi$ is the matrix of coefficients of the monomial matrix $\tilde{\xi}$. This sequence is an example of an Euler-type sequence (see [PT10] for some exactness properties). One can now consider the Buchsbaum-Rim complex associated to $\tilde{\xi}$ as has been done in [CT03]. As the maps $\eta$ and $\xi$ are related by Gale duality, it is straightforward to see that $\xi$ satisfies the uniformity condition of [CT03], Def. 4.6 (see also [Tch07], §2.4) if and only if the hyperplanes in $\mathcal{H}$ are in general position. In that case the Buchsbaum-Rim complex is a minimal resolution of $E_{\mathcal{H}}$. Then by Theorem 4.5 we get a nice combinatorial interpretation of the ranks of the graded parts of the Buchsbaum-Rim complex.

\subsection{Local cohomology}

In this subsection we will determine the local cohomologies $H_{x}^{i} E_{\mathcal{H}}$ of a hyperplane module $E_{\mathcal{H}}$ with respect to the torus fixed point $x$ in $\mathbf{A}_{\mathbb{K}}^{n}$. Let $\mathcal{G}$ be the gcd-lattice generated by the degrees of the nonzero graded Bass numbers of $E_{\mathcal{H}}$, as in Definition 3.42. It suffices to determine the dimensions $\operatorname{dim}\left(H_{x}^{i} E\right)_{\underline{d}}$ for every $\underline{d} \in \mathcal{G}$.

Lemma 4.10: Let $\underline{d} \in \mathcal{G}$ and denote $J=\left\{j \in[n] \mid \underline{d} \leq \underline{d}^{H_{j}}\right\} \subseteq[n]$. Moreover, denote $\mathcal{H}_{\underline{d}}$ the hyperplane arrangement generated by the $\left\{H_{j}\right\}_{j \in J}$. Then for every $k \geq 0$ the set $\left\{X \in \mathcal{H}_{k} \overline{\mid} \underline{d} \leq\right.$ $\underline{d}^{X}$ \} equals $\mathcal{H}_{\underline{d}} \cap \mathcal{H}_{k}$.

Proof. $\underline{d}^{V}$ is the unique maximal element in $\mathcal{G}$, thus $\underline{d} \leq \underline{d}^{V}$ for every $\underline{d} \in \mathcal{G}$. By the construction of subsection 3.2 we have $\underline{d}^{X}=\operatorname{gcd}\left\{\underline{d}^{H_{i}} \mid X \subseteq H_{i}\right\}$ for every $X \in \mathcal{H}$. Hence $\underline{d} \leq \underline{c}^{X}$ for every $X \in \mathcal{H}_{\underline{d}}$ and thus $\left\{X \in \mathcal{H}_{k} \mid \underline{d} \leq \underline{d}^{X}\right\}=\mathcal{H}_{\underline{d}} \cap \mathcal{H}_{k}$. 
According to Proposition 3.41, the $i$-th local cohomology at degree $\underline{d} \in \mathcal{G}$ is given by $\left(H_{x}^{i} E\right)_{\underline{d}} \cong H^{i+1}\left(B^{\bullet}, \underline{d}\right)$, where $B^{\bullet}, \underline{d}$ is a certain complex of vector spaces. With Lemma 4.10 we can describe this complex explicitly as follows. Consider the two subarrangements $\mathcal{H}_{\underline{d}}$ and $\mathcal{H}^{\underline{d}}$ of $\mathcal{H}$, where $\mathcal{H}_{\underline{d}}$ is generated by all $H \in \mathcal{H}$ such that $\underline{d} \leq \underline{d}^{H}$ and $\mathcal{H} \underline{d}$ is generated by the complementary set of hyperplanes in $\mathcal{H}$. In the following lemma we first collect the degenerate cases where $\mathcal{H}^{\underline{d}}$ contains at most one hyperplane.

Lemma 4.11: (i) If $\mathcal{H}^{\underline{d}}$ is generated by one hyperplane $H$ then $H^{i+1}\left(B^{\bullet}, \underline{d}\right)=0$ for all $i<n$ and $H^{n+1}\left(B^{\bullet}, \underline{d}\right)$ is naturally isomorphic to $V / H$.

(ii) If $\mathcal{H} \underline{\underline{d}}$ does not contain any hyperplane then $B^{i, \underline{d}}=0$ for $i \leq n$ and $H^{n+1}\left(B^{\bullet}, \underline{d}\right)$ is naturally isomorphic to $V$.

Proof. The statements of follow by inspection of the minimal resolution by coordinate arrangements of $\mathcal{H}$, where for (ii) we remark that the terms $B^{i, \underline{d}}$ for $i \leq n$ coincide with the minimal resolution of the hyperplane arrangement $\mathcal{H}$ restricted to $H$.

For the remaining case denote $\mathcal{B}_{j}^{\underline{d}}:=\mathcal{H}_{k} \backslash \mathcal{H}^{\underline{d}}$ for every $k \geq 0$. For every $k \leq n$ set $B^{k, \underline{d}}:=\bigoplus_{X \in \mathcal{B}_{n-k+1}^{\underline{d}}} X^{n-k}$ and $B^{n+1, \underline{d}}:=E$. Moreover, let $\eta^{k}$ be the restriction of $\phi_{n-k}$ to $\bigoplus_{X \in \mathcal{B}_{n-k+1}} X^{n-k}$. Then the complex $B^{\bullet}, \underline{d}$ has the following shape:

$$
0 \longrightarrow B^{n-t+1, \underline{d}} \stackrel{\eta^{n-t+1}}{\longrightarrow} \cdots \stackrel{\eta^{1}}{\longrightarrow} B^{n, \underline{d}} \stackrel{\eta^{n}}{\longrightarrow} B^{n+1, \underline{d}}=E \longrightarrow 0
$$

Its cohomoloy is described by the following proposition.

Proposition 4.12: Denote the length of a minimal resolution of $H^{\underline{d}}$ and assume that $H^{\underline{d}}$ is generated by at least two hyperplanes. Then $H^{i}\left(B^{\bullet}, \underline{d}\right)$ is zero for $i \neq n-t+1$ and $H^{n-t+1}\left(B^{\bullet}, \underline{d}\right)$ is isomorphic to the $t$-th syzygy of $V$ with respect to a minimal resolution of $\mathcal{H}^{\underline{d}}$. In particular the complex $B^{\bullet}, \underline{d}$ is exact iff $H^{\underline{d}}$ is a coordinate arrangement.

Proof. Assume that $\mathcal{H}^{\underline{d}}$ is essential and consider a minimal resolution of $\mathcal{H}^{\underline{d}}$ by coordinate arrangements $0 \rightarrow \mathcal{M}_{t} \rightarrow \cdots \rightarrow \mathcal{M}_{0} \rightarrow \mathcal{H}^{\underline{d}} \rightarrow 0$. By enumerating the hyperplanes in $\mathcal{H}_{\underline{d}}$ : $H_{1}, \ldots, H_{r}$, we can augment this resolution as follows. For every $0 \leq k<t$ and $i \geq 0$ we inductively define sets $\mathcal{S}_{k}^{0}=\mathcal{H}_{k}^{c}$ and $\mathcal{S}_{k}^{i}=\left\{X \cap H_{i} \mid X \in \mathcal{S}_{k+1}^{i-1}\right\}$ for $i>0$. We denote $\mathcal{A}_{k}^{i}:=\mathcal{S}_{k}^{i} \backslash$ $\mathcal{S}_{k}^{i-1}$ for $i>0$. Now for every $0 \leq k<t$ we set $\mathcal{M}_{k}^{0}:=\mathcal{M}_{k}$ and $\mathcal{M}_{k}^{i}:=\mathcal{M}^{i-1} \oplus \bigoplus_{X \in \mathcal{A}_{k}^{i}} X^{k-1}$ for $i>0$. Moreover, we define the differential among the $\mathcal{M}_{k}^{i}$ as the restrictions of the corresponding differentials $\phi_{k}$ of the minimal resolution of $\mathcal{H}$. We end up with the complex

$$
0 \longrightarrow \mathcal{M}_{t-1}^{r} \longrightarrow \cdots \longrightarrow \mathcal{M}_{0}^{r} \longrightarrow 0
$$

We can argue now analogous to the proof of Theorem 4.4 that our successive extensions lead to no new homology and the only homologies of this complex are $H^{0}\left(\mathcal{M}_{\bullet}^{r}\right) \cong V$ and $H^{t-1}\left(\mathcal{M}_{\bullet}^{r}\right)$ is isomorphic to $\mathcal{M}_{t}$. Then by passing to cohomological degrees and by forgetting about the underlying vector space arrangements we see that the complex $0 \rightarrow \mathcal{M}_{t-1}^{r} \rightarrow \cdots \rightarrow \mathcal{M}_{0}^{r} \rightarrow V \rightarrow$ 0 coincides with the complex (6) and our assertions follow.

In the case that $\mathcal{H}^{\underline{d}}$ is not essential with center $C$ assume that we are given a minimal resolution $0 \rightarrow \mathcal{M}_{t} \rightarrow \cdots \rightarrow \mathcal{M}_{0} \rightarrow \mathcal{H}^{\underline{d}} / C \rightarrow 0$ and assume we add another hyperplane $H$ to $\mathcal{H}^{\underline{d}}$. In the case that $H \supseteq C$, the center remains the same and we can apply our above discussion with respect to the vector space $V / C$. If $H \nsupseteq C$, then $H$ properly intersects all $X \in \mathcal{H}^{\underline{d}}$. In that case it is not difficult to see that the complex changes to $0 \rightarrow \mathcal{M}_{t} \rightarrow \cdots \rightarrow$ $\mathcal{M}_{1} \rightarrow \mathcal{M}_{0} \oplus H \cap X \rightarrow \mathcal{H} / C^{\prime}$, where $C^{\prime} \cong C / C \cap H$, i.e. only the 0 -th term of the resolution changes and immediately is compensated. 
For any $\mathcal{H}^{\underline{d}}$ with center $C \stackrel{d}{\underline{d}}$, we can associate to it the beta invariant $\beta^{\underline{d}}$ which is the beta invariant of the essential arrangement $\mathcal{H}^{\underline{d}} / C^{\underline{d}}$ in the sense of subsection 4.2. Its rank is given by $r^{\underline{d}}=\operatorname{dim} V / C \underline{d}$. If $\mathcal{H}^{\underline{d}}$ does not contain any hyperplane, then we use the convention that $C^{\underline{d}}=0$, hence $r^{\underline{d}}=\operatorname{dim} V$. Then Theorem 4.5 tells us that $\beta \underline{\underline{d}}$ equals the dimension of the $\left(r^{\underline{d}}-1\right)$ st syzygy of $V / C^{\underline{d}}$ with respect to a minimal resolution $\mathcal{H}^{\underline{d}}$ by coordinate arrangements. We can now show:

Theorem 4.13: Let $E=E_{\mathcal{H}}$ be a reflexive model of a hyperplane arrangement $\mathcal{H}$ in $V$. Denote $\mathcal{G}$ the gcd-lattice generated by the degrees of the Bass numbers of $E_{\mathcal{H}}$. For every $\underline{d} \in \mathcal{G}$ denote $\mathcal{H} \underline{d} \subset \mathcal{H}$ the hyperplane arrangement generated by those hyperplanes $H \in \mathcal{H}$ with $\underline{d} \not \leq \underline{d}^{H}, r \underline{d}$ its rank and $\beta \underline{\underline{d}}$ its beta invariant. Then for any $\underline{d} \in \mathcal{G}$ and any $\underline{c} \in \mathbb{Z}^{n}$ adjacent to $\underline{d}$ we have

$$
\operatorname{dim}\left(H_{x}^{i} E_{\mathcal{H}}\right)_{\underline{c}}= \begin{cases}\beta \underline{\underline{d}} & \text { if } i=n-r \underline{d}+1 \\ 0 & \text { else. }\end{cases}
$$

Proof. If $\mathcal{H}^{\underline{d}}$ contains at least two hyperplanes, this follows from Propositon 4.12, In the case that $\mathcal{H}$ contains less than two hyperplanes, this follows from Lemma 4.11, where we remark that, if $\mathcal{H}^{\underline{d}}$ is generated by one hyperplane $H$, then $C^{\underline{d}}=H$ and thus $\beta^{\underline{d}}=\operatorname{dim} V / H$.

\section{Codivisorial resolutions and maximal Cohen-Macaulay mod- ules}

In this section we will use the results of the previous sections to construct examples of $M$ graded MCM modules over rings $\mathbb{K}\left[\sigma_{M}\right]$, where $\sigma$ is a simplicial cone. Our strategy will be to start with a certain reflexive $S$-module $\hat{E}$ and to consider the graded structure of its minimal injective resolution. Now, if $S$ serves as a homogeneous coordinate ring for $\mathbb{K}\left[\sigma_{M}\right]$, we can derive conditions on $\sigma$ which tell us when $\hat{E}_{(0)}$ can be a MCM module over $\mathbb{K}\left[\sigma_{M}\right]$. More precisely, denote $x \in U_{\sigma}$ the torus fixed point and $\hat{x} \subseteq \mathbf{A}_{\mathbb{K}}^{n}$ its preimage under the surjection

$\mathbf{A}_{\mathbb{K}}^{n} \rightarrow U_{\sigma}$. Then we have $H_{x}^{i} \hat{E}_{(0)}=\left(H_{\hat{x}}^{i} \hat{E}\right)_{(0)}$ for any $i \in \mathbb{Z}$ (see Proposition [5.3). So, $\hat{E}_{(0)}$ is an MCM-module iff $\left(H_{\hat{x}}^{i} \hat{E}\right)_{(0)}=0$ for all $i<\operatorname{dim} \mathbb{K}\left[\sigma_{M}\right]$. If $\hat{E}$ is not free then we cannot expect that $H_{\hat{x}}^{i} \hat{E}$ will vanish for all such $i$. So the conditions we want to derive will tell us when $\sigma$ corresponds to an embedding $0 \rightarrow M \rightarrow \mathbb{Z}^{n}$ such that the nonvanishing degrees of $H_{\hat{x}}^{i} \hat{E}$ do not intersect $M$. Note that these kind of conditions will probably not lead to a classification of MCM modules over a fixed $\mathbb{K}\left[\sigma_{M}\right]$ with respect to, say, certain combinatorial invariants. As for the rank one case, any such direct approach would lead to rather complicated arithmetic conditions (see [Per11]), whose characterization is beyond the scope of this paper.

\subsection{Homogeneous coordinates and local cohomology}

Let $E$ be an $M$-graded $\mathbb{K}\left[\sigma_{M}\right]$-module ( $\sigma$ is not necessarily simplicial) and $F$ an $\mathbb{Z}^{n}$-graded $S$ module with $F_{(0)} \cong E$. Then any $\mathbb{Z}^{n}$-graded injective $I^{\bullet}$ resolution of $F$ induces a codivisorial resolution $I_{(0)}^{\bullet}$ of $E$. Of course, the shape of this resolution strongly depends on the choice of $F$. A standard choice is given by $F=\Gamma_{*} E$, where $\Gamma_{*} E$ denotes the graded tensor product $E \otimes_{\mathbb{K}\left[\sigma_{M}\right]}$ $S$. The $S$-module $\Gamma_{*} E$ has a natural $\mathbb{Z}^{n}$-grading which is given by $\Gamma_{*} E \cong \bigoplus_{\underline{c} \in \mathbb{Z}^{n}}\left(E \otimes_{\mathbb{K}\left[\sigma_{M}\right]}\right.$ $\left.S(\underline{c})_{(0)}\right)_{0}$. In particular, we have a right exact functor

$$
\Gamma_{*}: M-\mathbb{K}\left[\sigma_{M}\right]-\operatorname{Mod} \longrightarrow \mathbb{Z}^{n}-S \text {-Mod. }
$$

By observing that $\left(\Gamma_{*} E\right)_{0}=E$ for any $E$ it follows that the functor $(-)_{(0)}$ is essentially surjective (see also [Mus02, and [BC94] for the $\mathbb{Z}^{n}$-graded case).

In general, the module $\Gamma_{*} E$ might not be the best choice for a representative of $E$. For instance, even in good cases such as $E$ reflexive, $\Gamma_{*} E$ usually has torsion. However, as in this 
section we are interested exclusively in reflexive modules, we can give an alternative construction in this case.

Definition 5.1: Let $E$ be a finitely generated $M$-graded reflexive $\mathbb{K}\left[\sigma_{M}\right]$-module given by a vector space $\mathbf{E}$ and filtrations $E^{k}(i)$. Then we denote $\hat{E}$ the reflexive $\mathbb{Z}^{n}$-graded $S$-module associated to the same data.

In other words, as the fan of $\mathbf{A}_{\mathbb{K}}^{n}$ has the same number of rays as $\sigma_{M}$, we obtain $\hat{E}$ by simply reinterpreting the filtrations associated $E$. To see the effect, compare the graded pieces of $E$ and $\hat{E}$, respectively:

$$
E_{m}=\bigcap_{k \in[n]} E^{k}\left(l_{k}(m)\right) \quad \text { and } \quad \hat{E}_{\underline{c}}=\bigcap_{k \in[n]} E^{k}\left(c_{k}\right)
$$

for every $m \in M$ and every $\underline{c} \in \mathbb{Z}^{n}$. So, as not every intersection of the filtrations $E^{k}(i)$ must be realized as a graded component of $E$, we can consider $\hat{E}$ as the completion of $E$ with respect to intersections among the vector spaces in the filtrations. The following proposition shows that this construction also is functorially well-behaved.

Proposition 5.2: With $E$ and $\hat{E}$ as above we get:

(i) Passing from $E$ to $\hat{E}$ defines a fully faithful functor from the category of $M$-graded, finitely generated reflexive $\mathbb{K}\left[\sigma_{M}\right]$-modules to the category of $\mathbb{Z}^{n}$-graded, finitely generated reflexive $S$-modules.

(ii) $\hat{E}_{(0)} \cong E$.

(iii) In particular, the pair of functors $(-)_{(0)}, \hat{-}$ induces an equivalence of categories.

Proof. For (ii) we remark that vector space homomorphisms which are compatible with filtrations are also compatible with intersections of filtrations.

For (iii) observe that $E_{m}=\bigcap_{k \in[n]} E^{k}\left(l_{k}(m)\right)=\hat{E}_{L(m)}$ for every $m \in M$ (here, $L$ denotes the inclusion of $M$ into $\mathbb{Z}^{n}$ ).

Then (iii) follows directly from (ii) and (iii).

Once chosen a suitable $S$-module $F$ such that $E \cong F_{0}$, the following proposition tells us how we can obtain the local cohomologies for $E$ by computing those of $F$.

Proposition 5.3: Let $E$ be in $M-\mathbb{K}\left[\sigma_{M}\right]$-Mod and $F$ in $\mathbb{Z}^{n}-S$-Mod such that $E \cong F_{(0)}$. Moreover, let $V \subseteq U_{\sigma}$ be a torus invariant closed subvariety and $\hat{V}$ the preimage of $V$ in $\mathbf{A}_{\mathbb{K}}^{n}$. Then $H_{V}^{i} E \cong\left(H_{\hat{V}}^{i} F\right)_{(0)}$ for every $i \in \mathbb{Z}$.

Proof. Assume that we have an $\mathbb{Z}^{n}$-graded injective resolution $0 \rightarrow F \rightarrow I^{\bullet}$. By taking degree zero, we obtain a $M$-graded codivisorial resolution $0 \rightarrow E \rightarrow I_{(0)}^{\bullet}$. We obtain $H_{\hat{V}}^{i} F$ as the $i$-th cohomology of the complex $\Gamma_{\hat{V}} I^{\bullet}$ and, by Lemma 2.23 (iii), we obtain $H_{V}^{i} E$ as the $i$-th cohomology of the complex $\Gamma_{V} I_{(0)}^{\bullet}$. Using Lemma 2.23 (iii), we see that the complexes $\left(\Gamma_{\hat{V}} I^{\bullet}\right)_{(0)}$ and $\Gamma_{V} I_{(0)}^{\bullet}$ coincide.

\subsection{Vector space arrangements with trivial intersections}

Assume that $\sigma$ is simplicial but not smooth and $E$ an indecomposable reflexive $\mathbb{K}\left[\sigma_{M}\right]$-module such that $\hat{E}$ corresponds to a reflexive $S$-module of the type as considered in Examples 3.32 and 3.43, respectively. Using Proposition 5.3, we want to analyze the $\mathbb{Z}^{n}$-degrees of the local cohomology modules $H_{x}^{i} \hat{E}$, where $x$ is the torus fixed point in $U_{\sigma}$, with respect to the embedding of $M$ in $\mathbb{Z}^{n}$ in order to obtain conditions for the vanishing of $\left(H_{x}^{i} \hat{E}\right)_{(0)}$ for $i<d$. 
Using the notation of Example 3.43, we call, as in Definition 3.42, an element $\underline{c} \in \mathbb{Z}^{n}$ adjacent to $\underline{d}_{P}$, if $\underline{c} \leq \underline{d}_{P}$ and $\underline{c} \leq \leq \underline{d}_{Q}$ for all $Q \nsubseteq \subseteq P$. Then, for any $\underline{c}$ adjacent to some $\underline{d}_{P}$, we have $\left(H_{x}^{i} \hat{E}\right)_{\underline{c}}=\left(H_{x}^{i} \hat{E}\right)_{\underline{d}_{P}}$. We have seen in Example 3.43 that $\operatorname{dim}\left(H_{x}^{n-1} \hat{E}\right)_{\underline{d}_{P}}=\sum_{i \notin P} \operatorname{dim} V_{i}-$ $\operatorname{dim} \sum_{i \notin P} V_{i}$ for every $P \subseteq\{1, \ldots, t\}$. In other words, $\left(H_{x}^{n-1} \hat{E}\right)_{\underline{d}_{P}}$ vanishes iff the vector spaces $V_{i}$ indexed by the complement of $P$ form a linearly independent system of subvector spaces of $V$. We want to simplify our discussion by considering only cases where for any nonvanishing $\left(H_{x}^{n-1} \hat{E}\right)_{\underline{d}_{P}}$ there are only finitely many $\underline{c} \in \mathbb{Z}^{n}$ adjacent to $\underline{d}_{P}$.

Lemma 5.4: Assume that to any nonvanishing $b^{n-1}\left(\underline{d}_{P}\right)$ there are only finitely many $\underline{c} \in \mathbb{Z}^{n}$ adjacent to $\underline{d}_{P}$. Then $t=n$ and for any $i \in P$ the vector spaces $\left\{V_{j}\right\}_{j \neq i}$ form a linearly independent system of subvector spaces.

Proof. The assumption implies that $H_{x}^{n-1} \hat{E}$ is finite and therefore finitely generated. By [GD61, $\S$ VIII, Cor. 2.3, for $H_{x}^{n-1} \hat{E}$ being finitely generated it is necessary and sufficient that every localization $\hat{E}_{x_{i}}$ for $i \in[n]$ is a free $S_{x_{i}}$-module, which implies the assertion.

Corollary 5.5: Under the assumptions of Lemma 5.4. $\left(H_{x}^{n-1} \hat{E}\right)_{d_{\emptyset}}$ is the only nonvanishing graded Bass number in cohomological degree $n-1$.

Corollary 5.6: The vector spaces $V_{i}$ all have the same dimension $k$ and $\operatorname{dim} V=(n-1) k$.

Proof. First observe that $V_{i} \subseteq \sum_{j \neq i} V_{j}$ for every $i \in[n]$, as otherwise we could split $V_{i}=$ $V_{i}^{\prime}+V_{i}^{\prime \prime}$, where $V_{i}^{\prime}=V_{i} \cap \sum_{j \neq i} V_{j}$ and this way obtain a splitting of vector space arrangements $V \cong \sum_{j \neq i} V_{j} \oplus V_{i}^{\prime \prime}$. By Proposition 2.11 this would contradict the indecomposability of $\hat{E}$. With this observation, the assertion follows immediately.

Next we will see that indecomposability induces even stronger conditions.

Proposition 5.7: $\operatorname{dim} V_{i}=1$ for every $i \in[n]$. In particular, an indecomposable $M$-graded MCM-module satisfying the conditions of Lemma 5.4 has rank $n-1$.

Proof. By Corollaries 5.5 and 5.6, any choice of basis for $V_{1}, \ldots, V_{n-1}$ yields a basis for $V$. Now assume without loss generality that $V_{n}$ is in general position, i.e. the projection $\pi_{i}: V_{n} \rightarrow V_{i}$ is surjective for $1 \leq i<n$. So, a choice of basis $v_{1}, \ldots, v_{k}$ of $V_{n}$ induces a basis $\pi_{i}\left(v_{1}\right), \ldots, \pi_{k}$ of $V_{i}$ for every $1 \leq i<n$. Hence, for every $1 \leq j \leq k$, we get $W_{j}:=\sum_{i=1}^{n-1} \mathbb{K} \pi_{i}\left(v_{j}\right)$ with $\operatorname{dim} W_{j}=n-1$ and $v_{j} \in W_{j}$. Moreover, every $W_{j}$ is a filtered vector space with filtrations given by $\pi_{i}\left(v_{j}\right)$ for $1 \leq i<n$ and $v_{j}$, respectively, such that the decomposition $V \cong \bigoplus_{j=1}^{k} W_{j}$ is a decomposition of filtered vector spaces. By indecomposability of $E$ it follows that $k=1$.

The degrees adjacent to $\underline{d}_{\emptyset}$ form a cuboid lattice polytope given by $C=\left\{\underline{c} \in \mathbb{Z}^{n} \mid \underline{c} \leq \underline{d}_{\emptyset}\right.$ and $\underline{c} \not \leq \underline{d}_{\{i\}}$ for every $\left.i \in[n]\right\}$. We obtain the following general criterion.

Theorem 5.8: Under the assumptions of Lemma 5.4, $E$ is an $M C M$-module over $\mathbb{K}\left[\sigma_{M}\right]$ if and only if $C$ is contained in $\mathbb{Z}^{n} \backslash M$.

Theorem 5.8 remains somewhat vague as for a given $\sigma$, we leave open the problem of classifying the admissible cuboid regions $C$. The following theorem gives a precise statement for the case where $C$ consists of only one point. Let $C=\left\{\underline{d}_{\emptyset}=: \underline{d}\right\}$. We consider equivariant isomorphism class of reflexive $\mathbb{K}\left[\sigma_{M}\right]$-modules up to degree-shift by elements of $M$. In terms of filtrations this means that we consider filtrations $E^{k}(i)$ up to a simultaneous shift $E^{k}\left(i+l_{k}(m)\right)$ for every $k \in[n]$ by some $m \in M$. The following theorem classifies all isomorphism types up to degree-shift in terms of the divisor class group $A_{n-1}\left(U_{\sigma}\right)$. 
Theorem 5.9: Let $\sigma$ be a simplicial cone. Then there are, up degree-shift in $M$, precisely $\left|A_{n-1}\left(U_{\sigma}\right)\right|-1$ isomorphism classes of indecomposable MCM modules satisfying the assumptions of Lemma 5.4 such that $C$ consists of one element.

Proof. With notation as above, the possible $\underline{d}$ are classified by the cokernel of the short exact sequence $0 \rightarrow M \stackrel{L}{\rightarrow} \mathbb{Z}^{n} \rightarrow A_{n-1}\left(U_{\sigma}\right) \rightarrow 0$. The module $E$ is MCM iff $\underline{d}$ represents a nonzero element in $A_{n-1}\left(U_{\sigma}\right)$.

By Proposition [5.7, we can parameterize all modules $E$ satisfying above conditions by configurations of $n$ points in general position in $\mathbb{P} V \cong \mathbb{P}^{n-2}$ up to the action of $\mathrm{GL}_{\mathbb{K}}(V)$, which leaves us with precisely one isomorphism class.

Remark 5.10: Note that our conditions imply that $\operatorname{dim} U_{\sigma}>2$. It is well-known that equivariant reflexive modules over affine toric surfaces always split into a direct sum of modules of rank one.

Example 5.11: Consider the cone $\sigma$ in $\mathbb{Z}^{3}$ spanned by primitive vectors $l_{1}=(1,0,1), l_{2}=$ $(0,1,1)$, and $l_{3}=(-1,-1,1)$. Denote $\hat{E}$ a rank two reflexive $S$-module given by three lines $V_{1}, V_{2}, V_{2}$ in general position in $\mathbb{E} \cong \mathbb{K}^{2}$. We show that, up to degree-shift in $M$, there exist precisely five indecomposable equivariant isomorphism classes of MCM modules of rank two associated to this data.

Assume that $\underline{d}=\left(b_{1}, b_{2}, b_{3}\right)$ and $\underline{d}_{1}=\left(a_{1}, b_{2}, b_{3}\right), \underline{d}_{2}=\left(b_{1}, a_{2}, b_{3}\right), \underline{d}_{3}=\left(b_{1}, b_{2}, a_{3}\right)$ with $a_{k}<b_{k}$ for $1 \leq k \leq 3$. Its local cohomology is given by the cohomology of the following complex which is concentrated in cohomological degrees 2 and 3 :

$$
0 \longrightarrow \check{S}(-\underline{d}) \longrightarrow \check{S}\left(-\underline{d}_{1}\right) \oplus \check{S}\left(-\underline{d}_{2}\right) \oplus \check{S}\left(-\underline{d}_{3}\right) \longrightarrow 0 .
$$

Up to degree-shift, there are two isomorphism classes of MCM modules with $C=\{\underline{d}\}$ which are classified by

$$
0 \longrightarrow M \stackrel{L}{\longrightarrow} \mathbb{Z}^{3} \longrightarrow \mathbb{Z} / 3 \mathbb{Z} \longrightarrow 0 .
$$

For instance, we can choose $\underline{d} \in\{(1,1,0),(2,2,0)\}$.

A small computation shows that $C$ cannot have more than two elements. For the case that $C$ has two elements, we can find three more isomorphism classes, where $C$ can be represented by pairs $\left\{(1,1,0),(1,1,0)-e_{k}\right\}$, where for $1 \leq k \leq 3, e_{k}$ denote the standard basis vectors of $\mathbb{Z}^{3}$. Note that, as argued in the proof of Theorem 5.9, for any given $C$, there are no nontrivial equivariant moduli associated to $E$, we have precisely one isomorphism class.

Remark 5.12: Assume that we have an MCM-module $E$ as above with $C=\left\{\underline{d}=\left(d_{1}, \ldots, d_{n}\right)\right\}$. Then it follows from the general theory that its canonical dual $F:=\operatorname{Hom}(E, \omega)$ (here, $\omega$ denotes the canonical module of $\mathbb{K}\left[\sigma_{M}\right]$, which is isomorphic to $\left.S(\mathbf{1})_{(0)}\right)$ is MCM as well. However, it is interesting to verify this fact explicitly using our framework. The filtrations associated to $E$ are given for $k \in[n]$ by

$$
E^{k}(i)= \begin{cases}0 & \text { for } i<d_{k} \\ V_{k} & \text { for } i=d_{k} \\ V & \text { for } i>d_{k}\end{cases}
$$

Now, for its MCM-dual $F:=\operatorname{Hom}(E, \omega)$, we obtain the filtrations

$$
F^{k}(i)= \begin{cases}0 & \text { for } i<-d_{k}-1 \\ H_{k} & \text { for } i=-d_{k}-1 \\ V^{*} & \text { for } i \geq-d_{k}\end{cases}
$$


where $V^{*}=\operatorname{Hom}(V, \mathbb{K})$ and $H_{k}$ denotes the orthogonal complement of $V_{k}$ in $V^{*}$ with respect to the canonical pairing $V^{*} \times V \rightarrow \mathbb{K}$. The hyperplanes $H_{1}, \ldots, H_{n}$ form a hyperplane arrangement $\mathcal{H}$ in general position in $V^{*}$. The minimal resolution of $\hat{F}$ is given by

$$
0 \longrightarrow S\left(-\underline{c}^{V}\right) \longrightarrow \cdots \longrightarrow \bigoplus_{X \in \mathcal{H}_{k}} S\left(-\underline{c}^{X}\right) \longrightarrow \cdots \longrightarrow \bigoplus_{X \in \mathcal{H}_{1}} S\left(-\underline{c}^{X}\right) \longrightarrow \hat{F} \longrightarrow 0,
$$

where $\underline{c}^{X}=\left(c_{1}, \ldots, c_{n}\right)$ with $c_{k}=-d_{k}-1$ whenever $X \subseteq H_{k}$ and $c_{k}=-d_{k}$ else. In particular, $\underline{c}^{V}=-\underline{d}$. The local cohomology $H_{x}^{i} \hat{F}$ then is the cohomology of the following complex which is concentrated in cohomological degrees 2 to $n$ :

$$
0 \longrightarrow \check{S}\left(-\underline{f}^{V}\right) \longrightarrow \cdots \longrightarrow \bigoplus_{X \in \mathcal{H}_{k}} \check{S}\left(-\underline{f}^{X}\right) \longrightarrow \cdots \longrightarrow \bigoplus_{X \in \mathcal{H}_{1}} \check{S}\left(-\underline{f}^{X}\right) \longrightarrow 0
$$

where $f^{X}=\underline{c}^{X}+\mathbf{1}$ for every $X \in \mathcal{H}$. By Theorem 4.13, we have $H_{x}^{i} \hat{E}=0$ for $2<i<n$ and $\left(H_{x}^{2} \hat{E}\right)_{f^{V}} \cong \mathbb{K}$ for $\underline{c} \in C$ and $\left(H_{x}^{2} \hat{E}\right)_{\underline{c}}=0$, for $\underline{c} \neq \underline{f}^{V}$. We can conclude that $F$ is MCM iff $\underline{d}+\mathbf{1} \in \mathbb{Z}^{n} \backslash M$.

\section{References}

[AR89] M. Auslander and I. Reiten. The Cohen-Macaulay Type of Cohen-Macaulay Rings. Adv. Math., 73(1):1-23, 1989.

[ASS06] I. Assem, D. Simson, and A. Skowroński. Elements of the representation theory of associative algebras. Vol. 1., volume 65 of London Mathematical Society Student Texts. Cambridge University Press, 2006.

[BC94] V. V. Batyrev and D. A. Cox. On the Hodge structure of projective hypersurfaces in toric varieties. Duke Math. J., 75(2):293-338, 1994.

[BH98] W. Bruns and J. Herzog. Cohen-Macaulay Rings. Rev. Ed., volume 39 of Cambridge Studies in Advanced Mathematics. Cambridge University Press, 1998.

[Bre08] H. Brenner. Looking out for stable syzygy bundles. Advances in Mathematics, 219:401427, 2008.

[CT03] H. Charalambous and A. Tchernev. Free resolutions for multigraded modules: a generalization of Taylor's construction. Math. Res. Lett., 10(4):535-550, 2003.

[CZ09] G. Carlsson and A. Zomorodian. The Theory of Multidimensional Persistence. Discrete Comput. Geom., 42:71-93, 2009.

[Eis95] D. Eisenbud. Commutative Algebra with a View Toward Algebraic Geometry, volume 150 of Graduate Texts in Mathematics. Springer, 1995.

[Ful93] W. Fulton. Introduction to Toric Varieties. Princeton University Press, 1993.

[GD61] A. Grothendieck and J. A. Dieudonné. Eléments de Géométrie Algébrique II. Publ. Math. IHES, 8:5-222, 1961.

[GD71] A. Grothendieck and J. A. Dieudonné. Eléments de Géométrie Algébrique. I, volume 166 of Grundlehren der mathematischen Wissenschaften. Springer, 1971.

[GPW99] V. Gasharov, I. Peeva, and V. Welker. The lcm-lattice in monomial resolutions. Math. Res. Lett., 6(5-6):521-532, 1999. 
[GW78] S. Goto and K. Watanabe. On graded rings. II: $\mathbb{Z}^{n}$-graded rings. Tokyo J. Math., 1:237-261, 1978.

[GZ83] C. Greene and T. Zaslavsky. On the interpretation of Whitney numbers through arrangements of hyperplanes, zonotopes, non-Radon partitions, and orientations of graphs. Trans. Amer. Math. Soc., 280(1):97-126, 1983.

[HM03] D. Helm and E. Miller. Bass numbers of semigroup-graded local cohomology. Pacific J. of Math., 209(1):41-66, 2003.

[HM05] D. Helm and E. Miller. Algorithms for graded injective resolutions and local cohomology over semigroup rings. J. Symb. Comp., 39:373-395, 2005.

[Kly90] A. A. Klyachko. Equivariant Bundles on Toral Varieties. Math. USSR Izvestiya, 35(2):337-375, 1990.

[Kly91] A. A. Klyachko. Vector Bundles and Torsion Free Sheaves on the Projective Plane. Preprint Max Planck Institut für Mathematik, 1991.

[Knu08] K. P. Knudson. A Refinement of Multi-Dimensional Persistence. Homology, Homotopy and Applications, 10(1):259-281, 2008.

[Mil00] E. Miller. The Alexander Duality Functors and Local Duality with Monomial Support. J. Alg., 231:180-234, 2000.

[MS04] E. Miller and B. Sturmfels. Combinatorial Commutative Algebra, volume 227 of Graduate Texts in Mathematics. Springer, 2004.

[Mus02] M. Mustaţă. Vanishing Theorems on Toric Varieties. Tohoku Math. J., II. Ser., $54(3): 451-470,2002$.

[Nv04] C. Năstăsescu and F. van Oystaeyen. Methods of Graded Rings, volume 1836 of Lecture Notes in Mathematics. Springer, 2004.

[Oda88] T. Oda. Convex Bodies and Algebraic Geometry, volume 15 of Ergebnisse der Mathematik und ihrer Grenzgebiete. Springer, 1988.

[OT92] P. Orlik and H. Terao. Arrangements of Hyperplanes, volume 300 of Grundlehren der mathematischen Wissenschaften. Springer, 1992.

[Per04] M. Perling. Graded Rings and Equivariant Sheaves on Toric Varieties. Mathematische Nachrichten, 263-264:181-197, 2004.

[Per11] M. Perling. Divisorial Cohomology Vanishing on Toric Varieties. Documenta Mathematica, 16:209-251, 2011.

[PT10] M. Perling and G. Trautmann. Equivariant Primary Decomposition and Toric Sheaves. Manuscripta Math., 132(1-2):103-143, 2010.

[Röm01] T. Römer. Generalized Alexander duality and applications. Osaka J. Math., 38(2):469485, 2001.

[Sha69] R. Y. Sharp. The Cousin Complex for a Module over a Commutative Noetherian Ring. Math. Z., 112:340-356, 1969.

[Tch07] A. B. Tchernev. Representations of matroids and free resolutions for multigraded modules. Advances in Mathematics, 208:75-134, 2007. 
[TH86] N. V. Trung and L. T. Hoa. Affine semigroups and Cohen-Macaulay rings generated by monomials. Trans. Amer. Math. Soc., 298(1):145-167, 1986.

[Web07] P. Webb. An introduction to the representations and cohomology of categories. In Group representation theory, Lausanne, 2007, pages 149-173. EPFL Press, Lausanne, 2007.

[Yan01] K. Yanagawa. Sheaves on finite posets and modules over normal semigroup rings. $J$. Pure Appl. Algebra, 161:341-366, 2001.

[Yan03] K. Yanagawa. Stanley-Reisner rings, sheaves, and Poincaré-Verdier duality. Math. Res. Lett., 10:635-650, 2003.

[Yos90] Y. Yoshino. Cohen-Macaulay modules over Cohen-Macaulay rings, volume 146 of London Mathematical Society lecture note series. Cambridge University Press, 1990. 